\title{
Analysis of Kerr comb generation in silicon microresonators under the influence of two-photon absorption and fast free-carrier dynamics
}

\author{
P. Trocha $\odot,{ }^{1, *}$ J. Gärtner, ${ }^{2}$ P. Marin-Palomo $\odot,{ }^{1}$ W. Freude $\odot,{ }^{1}$ W. Reichel $\odot,{ }^{2}$ and C. Koos $\odot{ }^{1,3, \dagger}$ \\ ${ }^{1}$ Institute of Photonics and Quantum Electronics (IPQ), Karlsruhe Institute of Technology (KIT), 76131 Karlsruhe, Germany \\ ${ }^{2}$ Institute for Analysis (IANA), Karlsruhe Institute of Technology (KIT), 76131 Karlsruhe, Germany \\ ${ }^{3}$ Institute of Microstructure Technology (IMT), Karlsruhe Institute of Technology (KIT), 76131 Karlsruhe, Germany
}

(Received 31 March 2020; accepted 10 February 2021; published 22 June 2021)

\begin{abstract}
Kerr frequency comb generation relies on dedicated waveguide platforms that are optimized toward ultralow loss while offering comparatively limited functionality restricted to passive building blocks. In contrast to that, the silicon-photonic platform offers a highly developed portfolio of high-performance devices, but suffers from strong two-photon absorption (TPA) and subsequent free-carrier absorption (FCA) at near-infrared telecommunication wavelengths, thereby rendering Kerr comb generation a challenge. Here we present a model to investigate the impact of TPA and FCA on Kerr comb formation. Our model combines a modified version of the Lugiato-Lefever equation with a refined relation to precisely describe the fast space and time dependence of the free-carrier concentration along the circumference of the microresonator. Using this refined model, we derive conditions for modulation instability, in particular for necessary pump powers depending on TPA parameters and free-carrier lifetimes. We validate our analytical predictions by time integration and study the impact of fast free-carrier dynamics on Kerr comb formation. We find that silicon microresonators may be suitable for Kerr comb generation in the NIR, provided that the dwell time of the TPA-generated free carriers in the waveguide core is reduced by a reverse-biased $p-i-n$-junction and that the pump parameters are chosen appropriately.
\end{abstract}

DOI: 10.1103/PhysRevA.103.063515

\section{INTRODUCTION}

Generation of optical frequency combs in high- $Q$ Kerrnonlinear microresonators $[1,2]$ has the potential to unlock a wide range of applications such as timekeeping [3], frequency synthesis [4], optical communications [5], spectroscopy [6], and optical ranging [7,8]. Among different frequency comb states, dissipative Kerr solitons (DKSs) [9] are particularly attractive, offering broadband optical spectra with hundreds of phase-locked optical tones spaced by free spectral ranges of tens of gigahertz. As a key advantage in comparison to conventional comb sources built from discrete components, Kerr comb generators offer a small footprint and can be integrated into robust chip-scale photonic systems that lend themselves to cost-efficient mass production. So far, integrated optical Kerr comb sources have mostly been realized using specifically optimized silica and silicon nitride based waveguides that offer ultralow propagation loss down to $5.5 \mathrm{~dB} \mathrm{~m}^{-1}$ along with anomalous group-velocity dispersion [10,11], and that can bear high power levels. The functionality of these integration platforms, however, is still limited to merely passive

\footnotetext{
*philipp.trocha@kit.edu

†christian.koos@kit.edu
}

Published by the American Physical Society under the terms of the Creative Commons Attribution 4.0 International license. Further distribution of this work must maintain attribution to the author(s) and the published article's title, journal citation, and DOI. devices. In contrast to that, silicon photonics offers a highly developed portfolio of active and passive devices that are specifically geared toward operation at near-infrared (NIR) telecommunication wavelengths between 1200 and $1700 \mathrm{~nm}$. These devices can be reliably fabricated at low cost on large-area silicon substrates [12-14] and lend themselves to cointegration with electronic devices [15]. Expanding the silicon-photonic integration platform by monolithically integrated Kerr comb sources could have transformative impact regarding functionality, performance, footprint, and cost of comb-based optical systems. For wavelengths in the midinfrared, Kerr comb formation in silicon microresonators under the influence of three-photon absorption and free-carrier absorption was already experimentally demonstrated [16]. In contrast to that, at NIR telecommunication wavelengths, Kerr generation comb in silicon microresonators is impaired by two-photon absorption (TPA) and subsequent free-carrier absorption (FCA) and has so far only been investigated theoretically [17-19]. Interestingly, Ref. [17] predicts that generation of NIR Kerr combs should also be possible in silicon microresonators, provided that TPA-generated free carriers are removed from the Si waveguide core by reverse-biased $p-i-n$-junctions [20]. Specifically, the effective dwell times of the carriers in the waveguide core should be reduced from 1 to $5 \mathrm{~ns}[16,20-23]$ to, e.g., $100 \mathrm{ps}$, which is of the same order as the round-trip time of the soliton pulses in the cavity $[2,24]$. This conclusion, however, conflicts with the fact that Ref. [17] builds upon a formalism (see, e.g., in [21]) that ignores fast carrier dynamics and assumes a spatially uniform distribution of the free carriers along the microresonator circumference. 
In this paper, we expand the theoretical analysis of Kerr comb formation by including fast dynamics of TPA-generated free carriers. Building upon an analytical model that complements the Lugiato-Lefever equation (LLE $[25,26]$ ) by including TPA and dispersion anomalies of the ring resonances caused by avoided mode crossings, we introduce a description of the fast free-carrier dynamics based on a spatially nonuniform distribution along the microresonator circumference. Our approach allows us to describe the full spatiotemporal evolution of the free-carrier density within the ring and serves as a starting point for subsequent analysis of necessary conditions that must be fulfilled for achieving modulation instability (MI) and subsequent comb formation. Based on this analysis, we formulate simple conditions that must be fulfilled for achieving modulation instability and subsequent comb formation in dependence on the underlying TPA coefficient, the free-carrier lifetime, and FCA cross section. We describe the dependence of the MI threshold pump power on TPA and FCA parameters, and we find an upper limit for the TPA coefficient, above which comb formation is impossible even in the absence of FCA. The theoretical predictions are independently confirmed by numerical simulations that are based on time integration of the Lugiato-Lefever equation and the modified rate equation for the free carriers. While the model is general and can be broadly applied to different material platforms, we focus our investigation on silicon-photonic microresonators, in which the free-carrier dwell time can be artificially reduced by a reverse-biased $p-i-n$-junction that is built around the respective waveguide core. We find that Kerr comb generation in silicon microresonators can be achieved within technically realistic parameter ranges for free-carrier lifetime and pump power. We further develop and numerically validate a design for a silicon-photonic Kerr comb source that has a free spectral range (FSR) of $100 \mathrm{GHz}$ and a threshold pump power of $12 \mathrm{~mW}$ and that should even be suitable for dissipative Kerr-soliton (DKS) comb formation. It turns out that the incorporation of fast free-carrier dynamics changes the behavior of the optical waveforms in the microresonator to a notable degree, whereas the conditions for MI are essentially unaffected and remain consistent with the findings from simpler models [17-19].

\section{MODEL}

In our model, we describe the electric field $E(z, t)=$ $\operatorname{Re}\left[\underline{E}(z, t) e^{-i \omega_{\mathrm{p}} t}\right]$ in the Kerr-nonlinear microresonator (MR) by a carrier at the (angular) frequency $\omega_{\mathrm{P}}$ of the optical pump wave in combination with a complex slowly varying amplitude $\underline{E}(z, t)$ that depends on time $t$ and on the position $z$ along the resonator circumference. The presence of the strong pump leads to parametric gain for a pair of modes located symmetrically to both sides of the pump frequency $\omega_{\mathrm{P}}$. If the parametric gain for any of these modes exceeds the resonator losses, the corresponding mode amplitudes are amplified by resonant four-wave mixing, drawing energy from the pump wave. At the same time, the presence of a strong pump wave leads to generation of free carriers (FCs) through two-photon absorption (TPA). These carriers accumulate and lead to additional optical losses through free-carrier absorption (FCA). For silicon-photonic microresonators, the dwell time $\tau_{\text {eff }}$ and
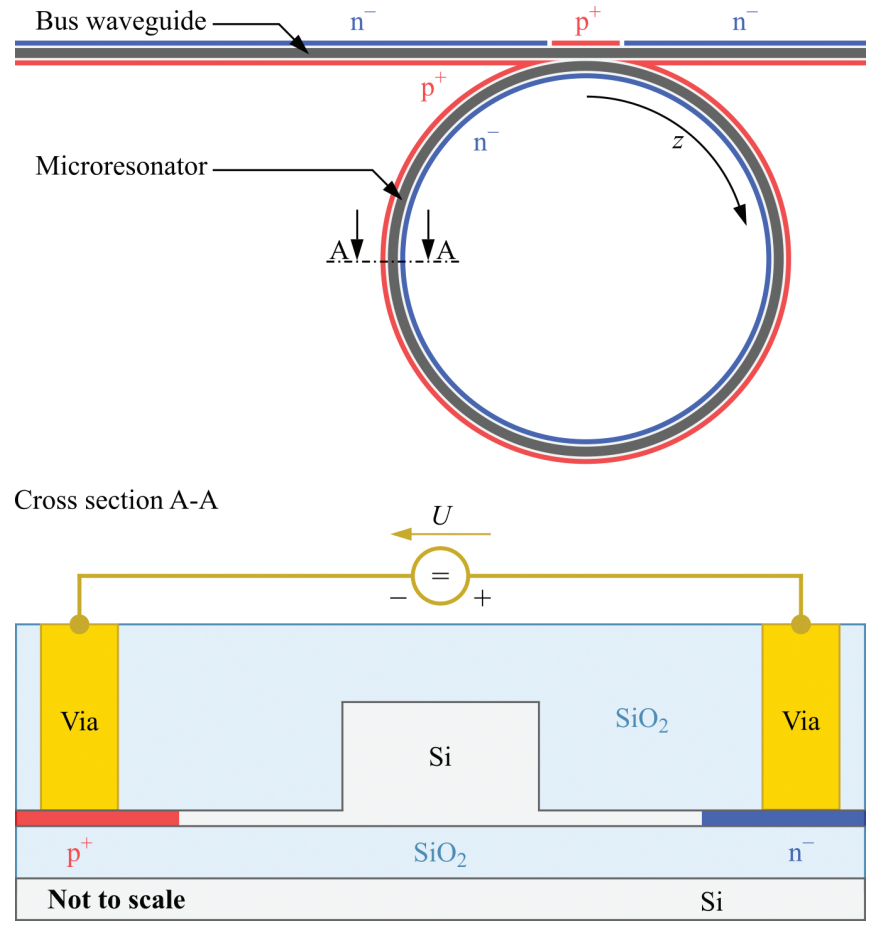

FIG. 1. Silicon-photonic microresonator as an example of a device suffering from both TPA and FCA. The bus waveguide as well as the resonator ring waveguide are connected to $p^{+}$-doped (red) and $n^{-}$-doped silicon ( $\mathrm{Si}$ ) regions (blue) that form a $p$ - $i$ - $n$-junction. This junction allows the reduction of the dwell time of free carriers by applying a reverse-bias voltage $U$ through contact pads and vias. At the coupling section between the bus and the ring waveguide, the doping is locally inverted from an $n^{-}$doping to a $p^{+}$doping next to the bus waveguide to ensure maximum free-carrier removal in the microresonator [28]. The geometrical dimensions of the waveguide cross section can be chosen such that the waveguide features anomalous group-velocity dispersion (GVD) at wavelengths near 1550nm, see, e.g., [29]. Further, the microresonator diameter can be chosen to achieve a specific free spectral range of, e.g., $f_{\mathrm{FSR}}=$ $100 \mathrm{GHz}$, corresponding to a round-trip time $t_{\mathrm{RT}}=f_{\mathrm{FSR}}^{-1}=10 \mathrm{ps}$. For details on the geometrical dimensions used in our simulations, see Sec. V.

the associated concentration $N_{\mathrm{c}}$ of the free carriers can be influenced by a reverse-biased $p$-i-n-junction [20,27]; see Fig. 1 . In the following, we consider the evolution of the field amplitude and of the free-carrier density over multiple cavity round-trip times $t_{\mathrm{RT}}=L / v_{\mathrm{g}}=f_{\mathrm{FSR}}^{-1}$, where $f_{\mathrm{FSR}}$ denotes the free spectral range of the cavity as defined by the perimeter $L$ and by the optical group velocity $v_{\mathrm{g}}=c / n_{\mathrm{g}}$ that is obtained at the pump frequency $\omega_{\mathrm{P}}$ in the absence of free carriers. The number of round trips is denoted by an integer $m$, and we introduce a long timescale ("slow" time variable) $t=m t_{\mathrm{RT}}$, which we consider to be continuous. At the same time, we model the evolution of the complex slowly varying amplitude $\underline{E}$ within the cavity using a short timescale ("fast" time variable) $\tau=t-z / v_{\mathrm{g}}$ that is retarded according to the position $z$ inside the cavity, thus accounting for the propagation of the optical amplitude $\underline{E}$ with optical group velocity $v_{\mathrm{g}}$. The slowly varying amplitude $\underline{E}(t, \tau)$ inside the resonator is modeled as a superposition of fields with complex envelopes $\underline{E}_{\Omega^{\prime}}(t)$, 
oscillating at equidistant angular frequencies which are offset from the pump frequency $\omega_{\mathrm{P}}=\omega_{0}$ by $\omega_{\Omega^{\prime}}=\Omega^{\prime}\left(2 \pi f_{\mathrm{FSR}}\right)$ $\left(\Omega^{\prime}=0, \pm 1, \pm 2, \pm 3, \cdots\right)$ and thus given as

$$
\underline{E}(t, \tau)=\sum_{\Omega^{\prime}} \underline{E}_{\Omega^{\prime}}(t) e^{i \omega_{\Omega^{\prime}} \tau}=\sum_{\Omega^{\prime}} \underline{E}_{\Omega^{\prime}}(t) e^{i 2 \pi \Omega^{\prime} \tau / t_{\mathrm{RT}}} .
$$

In contrast to the optical amplitude $\underline{E}(t, \tau)$, generated free carriers do not move inside the microresonator. Therefore, we model them on another, stationary fast timescale $\tau_{\mathrm{S}}=-z / v_{\mathrm{g}}$ to describe their spatial distribution $N_{\mathrm{c}}\left(t, \tau_{\mathrm{S}}\right)$ along the microresonator circumference. The stationary fast timescale $\tau_{\mathrm{S}}$ and its retarded counterpart are related by $\tau=$ $\tau_{\mathrm{S}}+t$. The optical field and the free-carrier density in the retarded time frame are transformed between the retarded and the static fast timescale via $\underline{E}(t, \tau)=\underline{E}\left(t, t+\tau_{\mathrm{S}}\right)$ and $N_{\mathrm{c}}(t, \tau-t)=N_{\mathrm{c}}\left(t, \tau_{\mathrm{S}}\right)$. The field obeys periodic boundary conditions, $\underline{E}(t, \tau)=\underline{E}\left(t, \tau+t_{\mathrm{RT}}\right)$. Disregarding temperature effects, self-steepening, higher-order dispersion, and higherorder multiphoton absorption, the LLE and the stationary FC equation read $[21,22]$

$$
\begin{aligned}
t_{\mathrm{RT}} \frac{\partial \underline{E}(t, \tau)}{\partial t}= & \sqrt{\kappa} \underline{E}_{\mathrm{in}}+\left[-\frac{\alpha_{i} L}{2}-\frac{\kappa}{2}-i \delta_{0}-i \frac{1}{2} \beta_{2} L \frac{\partial^{2}}{\partial \tau^{2}}\right. \\
& +i \hat{\Phi}_{\mathrm{AMC}}+\left(i \gamma L-\frac{\beta_{\mathrm{TPA}} L}{2 A_{\mathrm{eff}}}\right)|\underline{E}(t, \tau)|^{2} \\
& \left.-\frac{\sigma \Gamma_{\mathrm{c}} L}{2}(1+i \mu) N_{\mathrm{c}}(t, \tau-t)\right] \underline{E}(t, \tau), \\
\frac{\partial N_{\mathrm{c}}\left(t, \tau_{\mathrm{S}}\right)}{\partial t}= & \frac{\beta_{\mathrm{TPA}}}{2 \hbar \omega_{\mathrm{P}}} \frac{\left|\underline{E}\left(t, \tau_{\mathrm{S}}+t\right)\right|^{4}}{A_{\mathrm{eff}}^{2}}-\frac{N_{\mathrm{c}}\left(t, \tau_{\mathrm{S}}\right)}{t_{\mathrm{eff}}} .
\end{aligned}
$$

In these relations, the quantity $\underline{E}_{\text {in }}$ denotes the electric field amplitude of the pump with power $P_{\text {in }}=\left|E_{\text {in }}\right|^{2}$ in the bus waveguide (see Fig. 1), $\kappa$ describes the power coupling to the microresonator, and $\alpha_{i}$ (unit $\mathrm{m}^{-1}$ ) is the waveguide power loss coefficient inside the MR. The resonator parameters are assumed to be the same for all complex envelopes $\underline{E}_{\Omega^{\prime}}(t)$, unless specified otherwise. The detuning $\delta_{0}=\left(\omega_{\mathrm{R}}-\omega_{\mathrm{P}}\right) t_{\mathrm{RT}}$ corresponds to the offset of the pump frequency $\omega_{\mathrm{P}}$ from the resonance frequency $\omega_{\mathrm{R}}$ of the pumped mode. The coefficient $\beta_{2}$ describes the second-order dispersion of the cavity. The nonlinearity parameter of the resonator waveguide is denoted by $\gamma=\omega_{\mathrm{P}} c^{-1} n_{2} A_{\text {eff }}^{-1}$ (unit $\mathrm{m}^{-1} \mathrm{~W}^{-1}$ ) with the nonlinear Kerr coefficient $n_{2}$ (unit $\mathrm{m}^{2} \mathrm{~W}^{-1}$ ), the vacuum speed of light $c$, and the effective mode-field area $A_{\text {eff }}$. The quantity $\beta_{\text {TPA }}$ denotes the two-photon absorption coefficient, $\sigma$ is the free-carrier absorption cross section, and $\mu$ the free-carrier dispersion parameter which describes the influence of $\mathrm{FC}$ on the real part of the refractive index. The reduced Planck constant is $\hbar$. The model additionally includes a field confinement factor $\Gamma_{\mathrm{c}}$, which accounts for the fact that only a fraction of the optical mode field experiences the attenuation and dispersion by FCs generated in the resonator waveguide [30]. Since we consider only modes for which the field is strongly confined to the waveguide, we may assume $\Gamma_{\mathrm{c}} \approx 1$, whereas other waveguide designs, e.g., slot waveguides with a nonlinear organic cladding [31,32], may lead to values of $\Gamma_{\mathrm{c}}$ that are significantly smaller than 1 . Finally, we include the possibility of local resonance shifts $\delta \omega_{\Omega^{\prime}}$ caused by avoided mode crossings (AMCs) [33-35]. These resonance shifts lead to additional phase shifts $\delta \omega_{\Omega^{\prime}} t_{\mathrm{RT}}$ for the respective electric field envelopes $E_{\Omega^{\prime}}(t)$. The impact of AMCs can hence be described by an operator $\hat{\Phi}_{\mathrm{AMC}}$ acting on the envelope field $\underline{E}(t, \tau)$ of Eq. (1),

$$
\begin{aligned}
\hat{\Phi}_{\mathrm{AMC}} \underline{E}(t, \tau) & =\sum_{\tilde{\Omega}}\left[\delta \omega_{\tilde{\Omega}} \exp \left(i 2 \pi \tilde{\Omega} \tau / t_{\mathrm{RT}}\right) \int_{0}^{t_{\mathrm{RT}}} \underline{E}\left(t, \tau_{1}\right) \exp \left(-i 2 \pi \tilde{\Omega} \tau_{1} / t_{\mathrm{RT}}\right) \mathrm{d} \tau_{1}\right] \\
& =\sum_{\tilde{\Omega}, \Omega^{\prime}}\left[\delta \omega_{\tilde{\Omega}} t_{\mathrm{RT}} \underline{E}_{\Omega^{\prime}}(t) \exp \left(i 2 \pi \tilde{\Omega} \tau / t_{\mathrm{RT}}\right) \delta_{\tilde{\Omega}, \Omega^{\prime}}\right] \\
& =\sum_{\Omega^{\prime}}\left[\delta \omega_{\Omega^{\prime}} t_{\mathrm{RT}} \underline{E}_{\Omega^{\prime}}(t) \exp \left(i 2 \pi \Omega^{\prime} \tau / t_{\mathrm{RT}}\right)\right],
\end{aligned}
$$

where $\delta_{\tilde{\Omega}, \Omega^{\prime}}$ denotes the Kronecker delta. In the absence of AMCs, all resonance shifts $\delta \omega_{\Omega^{\prime}}$ are zero and thus do not lead to any additional phase shifts $\delta \omega_{\Omega^{\prime}} t_{\mathrm{RT}}$ for the electric field envelopes $E_{\Omega^{\prime}}(t)$. In the following, we simplify Eq. (2) by assuming critical coupling, i.e., $\alpha_{i} L=\kappa$. For normalization, we multiply Eq. (2) with $\sqrt{\gamma n_{\mathrm{g}}^{2} /\left(\alpha_{i}^{3} t_{\mathrm{RT}}^{2} c^{2}\right)}$ and Eq. (3) with $n_{2} \hbar \omega_{\mathrm{P}}^{2} /\left(\alpha_{i}^{2} c\right)$. We introduce the normalized quantities specified in Table I and reformulate Eqs. (2)-(4):

$$
\begin{gathered}
\frac{\partial \underline{a}\left(t^{\prime}, \tau^{\prime}\right)}{\partial t^{\prime}}=\sqrt{F}+\left[-1-i \zeta+i d \frac{\partial^{2}}{\partial \tau^{\prime 2}}+i \hat{\Phi}_{\mathrm{AMC}}^{\prime}+(i-r)\left|\underline{a}\left(t^{\prime}, \tau^{\prime}\right)\right|^{2}-\sigma^{\prime}(1+i \mu) N_{\mathrm{c}}^{\prime}\left(t^{\prime}, \tau^{\prime}-t^{\prime} v^{\prime}\right)\right] \underline{a}\left(t^{\prime}, \tau^{\prime}\right), \\
\frac{\partial N_{\mathrm{c}}^{\prime}\left(t^{\prime}, \tau_{\mathrm{S}}^{\prime}\right)}{\partial t^{\prime}}=r\left|\underline{a}\left(t^{\prime}, \tau_{\mathrm{S}}^{\prime}+t^{\prime} v^{\prime}\right)\right|^{4}-\frac{N_{\mathrm{c}}^{\prime}\left(t^{\prime}, \tau_{\mathrm{S}}^{\prime}\right)}{t_{\mathrm{eff}}^{\prime}}, \\
\hat{\Phi}_{\mathrm{AMC}}^{\prime} \underline{a}\left(t^{\prime}, \tau^{\prime}\right)=\sum_{\tilde{\Omega}} \frac{\phi_{\tilde{\Omega}}}{2 \pi} \int_{0}^{2 \pi} \underline{a}\left(t^{\prime}, \tau_{1}\right) \exp \left[-i \tilde{\Omega} \tau_{1}\right] d \tau_{1} \exp \left[i \tilde{\Omega} \tau^{\prime}\right] .
\end{gathered}
$$

In Eqs. (5) and (6), we have introduced the normalized group velocity $v^{\prime}=2 \pi n_{\mathrm{g}} /\left(t_{\mathrm{RT}} \alpha_{i} c\right)$ to describe the speed at which the normalized optical field $\underline{a}\left(t^{\prime}, \tau^{\prime}\right)=\underline{a}\left(t^{\prime}, \tau_{\mathrm{S}}^{\prime}+t^{\prime} v^{\prime}\right)$ propagates on the normalized stationary fast timescale $\tau_{\mathrm{S}}^{\prime}$. In the same manner, the FC density in the normalized retarded fast time frame is described by $N_{\mathrm{c}}^{\prime}\left(t^{\prime}, \tau^{\prime}-t^{\prime} v^{\prime}\right)=N_{\mathrm{c}}^{\prime}\left(t^{\prime}, \tau_{\mathrm{S}}^{\prime}\right)$. 
TABLE I. Normalized parameters and physical quantities.

\begin{tabular}{|c|c|c|c|}
\hline Slow time & $t^{\prime}=\alpha_{i} L t / t_{\mathrm{RT}}=t \alpha_{i} c / n_{\mathrm{g}}$ & Detuning & $\zeta=\delta_{0} n_{\mathrm{g}} /\left(\alpha_{i} t_{\mathrm{RT}} c\right)$ \\
\hline Fast time (retarded) & $\tau^{\prime}=2 \pi \tau / t_{\mathrm{RT}}$ & Phase shift operator & $\hat{\Phi}_{\mathrm{AMC}}^{\prime}=\hat{\Phi}_{\mathrm{AMC}} n_{\mathrm{g}} /\left(\alpha_{i} t_{\mathrm{RT}} c\right)$ \\
\hline Fast time (stationary) & $\tau_{\mathrm{S}}^{\prime}=2 \pi \tau_{\mathrm{S}} / t_{\mathrm{RT}}$ & Phase shifts & $\phi_{\Omega^{\prime}}=\delta \omega_{\Omega^{\prime}} n_{\mathrm{g}} /\left(\alpha_{i} c\right)$ \\
\hline Normalized group velocity & $v^{\prime}=2 \pi n_{\mathrm{g}} /\left(t_{\mathrm{RT}} \alpha_{i} c\right)$ & Dispersion & $d=-2 \beta_{2} \pi^{2} /\left(\alpha_{i} t_{\mathrm{RT}}^{2}\right)$ \\
\hline Optical field & $\underline{a}\left(t^{\prime}, \tau^{\prime}\right)=\sqrt{\gamma / \alpha_{i}} \underline{E}(t, \tau)$ & TPA parameter & $r=c \beta_{\mathrm{TPA}} /\left(2 \omega_{\mathrm{P}} n_{2}\right)$ \\
\hline Free-carrier density & $N_{\mathrm{c}}^{\prime}\left(t^{\prime}, \tau^{\prime}\right)=\hbar \omega^{2} n_{2} /\left(\alpha_{i} n_{\mathrm{g}}\right) N_{\mathrm{c}}(t, \tau)$ & FC dwell time & $t_{\mathrm{eff}}^{\prime}=t_{\mathrm{eff}} \alpha_{i} c / n_{\mathrm{g}}$ \\
\hline Pump field & $\sqrt{F}=\sqrt{\gamma P_{\text {in }} n_{\mathrm{g}} /\left(c \alpha_{i}^{2} t_{\mathrm{RT}}\right)}$ & FC cross section & $\sigma^{\prime}=\sigma \Gamma_{\mathrm{c}} n_{\mathrm{g}} /\left(2 n_{2} \hbar \omega^{2}\right)$ \\
\hline
\end{tabular}

\section{INVESTIGATION OF MODULATION INSTABILITY CONSIDERING FAST-TIME FREE-CARRIER DYNAMICS}

To investigate the circumstances under which modulation instability can occur when pumping the resonator, we need to know whether any pair of resonator modes experiences a sufficiently high parametric gain to overcome the total loss in the resonator. This loss includes linear propagation loss and coupling loss, which are equal for critical coupling, $\alpha_{i} L=\kappa$, and which are expressed by -1 in Eq. (5), two-photon absorption, expressed by $-r\left|\underline{a}\left(t^{\prime}, \tau^{\prime}\right)\right|^{2}$ in Eq. (5), and free-carrier absorption, expressed by $-\sigma^{\prime} N_{\mathrm{c}}^{\prime}\left(t^{\prime}, \tau^{\prime}-t^{\prime} v^{\prime}\right)$ in Eq. (5), where the normalized density of accumulated carriers strongly depends on the carrier dwell time $t_{\text {eff }}$ and its normalized counterpart $t_{\text {eff }}^{\prime}$, see Eq. (6). For exploring modulation instability, we use an ansatz for the normalized optical resonator field $\underline{a}\left(t^{\prime}, \tau^{\prime}\right)$, consisting of a strong field $\underline{a}_{0}$ in the pumped resonator mode (normalized power $A=\left|\underline{a}_{0}\right|^{2}$ ) and a pair of weak fields $\underline{a}_{ \pm \Omega}$ ("side bands" for short) in resonator modes, which are offset from the pump frequency $\omega_{\mathrm{P}}$ by $\omega_{ \pm \Omega}= \pm \Omega\left(2 \pi f_{\mathrm{FSR}}\right)\left(\Omega \in \mathbb{N}_{+}\right)$[36]. The amplitude of these side bands may change, which is expressed by a complex normalized gain rate $\underline{\lambda}=\lambda+i \lambda_{i}, \lambda, \lambda_{i} \in \mathbb{R}$. This leads to a three-wave ansatz of the form

$$
\begin{aligned}
\underline{a}\left(t^{\prime}, \tau^{\prime}\right)= & \underline{a}_{0}+\underline{\hat{a}}_{+\Omega} e^{\lambda t^{\prime}} e^{i \lambda_{i} t^{\prime}} e^{i \Omega \tau^{\prime}} \\
& +\underline{\hat{a}}_{-\Omega} e^{\lambda t^{\prime}} e^{-i \lambda_{i} t^{\prime}} e^{-i \Omega \tau^{\prime}},\left|\underline{\hat{a}}_{ \pm \Omega}\right| \ll\left|\underline{\hat{a}}_{0}\right| .
\end{aligned}
$$

In the stationary fast time frame, this ansatz reads

$$
\begin{aligned}
\underline{a}\left(t^{\prime}, \tau_{\mathrm{S}}^{\prime}+t^{\prime} v^{\prime}\right)= & \underline{a}_{0}+\underline{\hat{a}}_{+\Omega} e^{\lambda t^{\prime}} e^{i \lambda_{i} t^{\prime}} e^{i \Omega\left(\tau_{\mathrm{S}}^{\prime}+t^{\prime} v^{\prime}\right)} \\
& +\underline{\hat{a}}_{-\Omega} e^{\lambda t^{\prime}} e^{-i \lambda_{i} t^{\prime}} e^{-i \Omega\left(\tau_{\mathrm{S}}^{\prime}+t^{\prime} v^{\prime}\right)} .
\end{aligned}
$$

We assume that the side-band amplitudes $\underline{\hat{a}}_{ \pm \Omega}$ are initially much smaller than the amplitude of the pumped mode $\underline{a}_{0},\left|\underline{\hat{a}}_{ \pm \Omega}\right| \ll\left|\underline{a}_{0}\right|$, such that we can treat them as a weak perturbation by linearizing Eqs. (5) and (6) in $\underline{\hat{a}}_{+\Omega}, \underline{\hat{a}}_{-\Omega}$ about the strong field of the pumped mode $\underline{a}_{0}$. Inserting Eq. (8) into the linearized version of Eq. (5) and inserting Eq. (9) into Eq. (6) allows us to derive an expression for the normalized gain rate $\underline{\lambda}(\Omega)$; see Appendix A. Modulation instability occurs for $\operatorname{Re}\{\underline{\lambda}(\Omega)\}=\lambda(\Omega)>0$, in which case the side-band amplitudes at $\pm \Omega$ grow exponentially with time. The initial field oscillates with $\pm \Omega$ with respect to the normalized fast time $\tau^{\prime}$, and it experiences a phase shift $\pm \lambda_{i} t^{\prime}$ with normalized slow time $t^{\prime}$.

To identify resonator and pump parameters for which modulation instability can occur, we first derive an expression for $\underline{\lambda}$ in terms of these parameters. To this end, we first solve the differential equation for the normalized free-carrier density, Eq. (6), and then substitute the resulting relation for $N_{\mathrm{c}}^{\prime}\left(t^{\prime}, \tau_{\mathrm{S}}^{\prime}\right)$ in Eq. (5). In this step, we assume the modulation instability to be weak, i.e., $|\lambda| \ll 1 / t_{\text {eff }}^{\prime}$. Since we are looking at the onset of MI just above its threshold, the gain parameter $\lambda$ is barely larger than zero and is smaller than the inverse of the free-carrier lifetime, i.e., $|\lambda| \ll 1 / t_{\text {eff }}^{\prime}$. The latter assumption is justified by the fact that, for proper choice of reverse-bias conditions, the carrier dwell time is of the order of the cavity round-trip time, whereas the MI side bands build up slowly over tens of cavity round trips if the resonator is operated slightly above its MI threshold. Further details on the derivation and on the underlying approximations can be found in Appendix A. We further assume the imaginary part $\lambda_{i}$ of the gain parameter to be small, in particular $\left|\lambda_{i}\right| \ll \Omega v^{\prime}$, which is justified by the fact that MI side bands will occur in close spectral vicinity of a ring resonance; for details see Appendix A. With an expression for the free-carrier density at hand [see Eqs. (A8) and (A9) of Appendix A], we substitute Eq. (8) in Eq. (5) and neglect all small second-order products of the form $\underline{\hat{a}}_{ \pm \Omega}^{2},\left|\underline{\hat{a}}_{ \pm \Omega}\right|^{2}, \underline{\hat{a}}_{+\Omega} \underline{\hat{a}}_{-\Omega}$. We then solve the resulting equation for $\underline{\lambda}$ (see Appendix A), and obtain two complex solutions $\underline{\lambda}_{ \pm}=\lambda_{ \pm}+i \lambda_{ \pm, i}$. From these solutions, we select the one for which the real part $\lambda_{+}$can assume positive values, corresponding to modulation instability,

$$
\begin{aligned}
& \lambda(\Omega)=-1-2 r A-\sigma^{\prime} r t_{\mathrm{eff}}^{\prime} \frac{3+\left(\Omega t_{\mathrm{eff}}^{\prime} v^{\prime}\right)^{2}}{1+\left(\Omega t_{\mathrm{eff}}^{\prime} v^{\prime}\right)^{2}} A^{2}+\operatorname{Re}\{\underline{\Delta}\} \text { with } \operatorname{Re}\{\underline{\Delta}\}>0, \\
& \lambda_{i}(\Omega)=\frac{\phi_{+\Omega}-\phi_{-\Omega}}{2}+2 \frac{\sigma^{\prime} r t_{\mathrm{eff}}^{\prime} \Omega t_{\mathrm{eff}}^{\prime} v^{\prime}}{1+\left(\Omega t_{\mathrm{eff}}^{\prime} v^{\prime}\right)^{2}} A^{2}+\operatorname{Im}\{\underline{\Delta}\}, \\
& \Delta=\left\{A^{2}\left[r+\frac{2 \sigma^{\prime} r t_{\mathrm{eff}}^{\prime}}{1+\left(\Omega t_{\mathrm{eff}}^{\prime} v^{\prime}\right)^{2}} A-i \frac{2 \sigma^{\prime} r t_{\mathrm{eff}}^{\prime} \Omega t_{\mathrm{eff}}^{\prime} v^{\prime}}{1+\left(\Omega t_{\mathrm{eff}}^{\prime} v^{\prime}\right)^{2}} A\right]^{2}+A^{2}\left[1-\frac{2 \sigma^{\prime} r t_{\mathrm{eff}}^{\prime} \mu}{1+\left(\Omega t_{\mathrm{eff}}^{\prime} v^{\prime}\right)^{2}} A+i \frac{2 \sigma^{\prime} r t_{\mathrm{eff}}^{\prime} \mu \Omega t_{\mathrm{eff}}^{\prime} v^{\prime}}{1+\left(\Omega t_{\mathrm{eff}}^{\prime} v^{\prime}\right)^{2}} A\right]^{2}\right. \\
& \left.-\left[\zeta+d \Omega^{2}-\frac{\phi_{+\Omega}+\phi_{-\Omega}}{2}-2 A+\sigma^{\prime} r t_{\mathrm{eff}}^{\prime} \mu \frac{3+\left(\Omega t_{\mathrm{eff}}^{\prime} v^{\prime}\right)^{2}}{1+\left(\Omega t_{\mathrm{eff}}^{\prime} v^{\prime}\right)^{2}} A^{2}-i \frac{2 \sigma^{\prime} r t_{\mathrm{eff}}^{\prime} \mu \Omega t_{\mathrm{eff}}^{\prime} v^{\prime}}{1+\left(\Omega t_{\mathrm{eff}}^{\prime} v^{\prime}\right)^{2}} A^{2}\right]^{2}\right\}^{1 / 2} .
\end{aligned}
$$


Both the real part $\lambda$ and the imaginary part $\lambda_{i}$ of the gain parameter show a dependence on the normalized power $A$ of the pumped mode. For a given normalized pump power $F$, the normalized power $A$ in the pumped mode can be determined by evaluating the expression

$$
F=[\left(1+r A+r t_{\mathrm{eff}}^{\prime} \sigma^{\prime} A^{2}\right)^{2}+\underbrace{\left(A-\zeta+\phi_{0}-\sigma^{\prime} r t_{\mathrm{eff}}^{\prime} \mu A^{2}\right)^{2}}_{(*)}] A
$$

A derivation of Eq. (11) can be found in Appendix A.

\section{PARAMETER RANGES OF TPA, FCA, AND PUMP POWER LEADING TO MODULATION INSTABILITY}

Using Eqs. (10) and (11) for a specific parameter set $\left(r, \sigma^{\prime}, t_{\mathrm{eff}}^{\prime}, \mu, d, v^{\prime}, \phi_{\tilde{\Omega}}\right)$ and for specific operating conditions $(F, \zeta)$, we can determine whether MI can occur and at which side bands $\Omega^{\prime}= \pm \Omega$ it will happen. To reduce the complexity of the evaluation, we simplify Eq. (10) by considering technically relevant sets of normalized parameters $\left(r \approx 1, \sigma^{\prime} \approx 5, t_{\mathrm{eff}}^{\prime} \approx 0.05-0.5, \mu \approx 10, v^{\prime} \approx 200\right.$, $F \approx 10$ ) and by assuming that the side modes, for which MI will occur, are not affected by AMC, i.e., $\phi_{+\Omega}=\phi_{-\Omega}=0$. The normalized parameters are obtained using the relations in Table I in combination with the physical parameters listed in Tables II and III; see Appendix G. Specifically, we assume that TPA-generated free carriers are removed by a reverse-biased $p$ - $i$ - $n$-junction and thus have a small dwell time $t_{\text {eff }} \approx(12-100)$ ps [20], which is of the same order of magnitude as the cavity round-trip time. For estimating the normalized intracavity power $A$ of the pumped mode, we use Eq. (11) and assume that a detuning $\zeta=A+\phi_{0}-\sigma^{\prime} r t_{\text {eff }}^{\prime} \mu A^{2}$ is chosen for optimized power transfer from the pump $F$ to the pumped resonator mode, which makes the expression (*) $\left(A-\zeta+\phi_{0}-\sigma^{\prime} r t_{\text {eff }}^{\prime} \mu A^{2}\right)^{2}$ on the right-hand-side of Eq. (11) vanish. With the above-mentioned parameters, Eq. (11) can then be written as $F=\left(1+r A+\sigma^{\prime} r t_{\text {eff }}^{\prime} A^{2}\right)^{2} A$, leading to $A \approx 1$ for a large range of technically relevant normalized pump powers $F$ between 1 and 100 . Note that the following investigation aims at identifying the dominant terms in Eq. (10) and that the symbol " $\approx "$ is to be understood as an order of magnitude quantification rather than as an approximate equality.

To identify the side bands at which MI will occur first, we need to find values of the side-band offset $\Omega$ that maximize the gain rate $\lambda$. To this end, we simplify Eq. (10) by reducing it to its dominant terms, assuming that the offset of the MIgenerated side bands from the pump is of the order of $\Omega \approx 10$. With the above-mentioned parameters, this leads to

$$
\lambda(\Omega)=-1-r\left(2+\sigma^{\prime} t_{\mathrm{eff}}^{\prime} A\right) A+\operatorname{Re}\left\{\sqrt{A^{2}\left(r^{2}+1\right)-\left(\zeta+d \Omega^{2}-2 A+\sigma^{\prime} r t_{\mathrm{eff}}^{\prime} \mu A^{2}\right)^{2}}\right\}
$$

see Appendix B for details. This formula was also obtained in a previous study neglecting the fast-time dynamics of free carriers [17]. We therefore conclude that the fast-time dynamics of $N_{\mathrm{c}}^{\prime}$ have negligible impact on MI. This can be understood by the fact that, at the onset of MI, the optical power is still uniformly distributed along the circumference of the resonator, such that fast carrier dynamics on a timescale smaller than the round-trip time do not play a big role. In the absence of FCA, i.e., for $\sigma^{\prime}=0$, Eq. (12) is consistent with results from previous studies investigating the impact of TPA on modulation stability $[18,19]$. Similarly, maintaining only FCA-related terms in Eq. (12) leads to relations that have previously been derived for resonators impaired by free carriers only [19]. We simplify the further analysis, by treating $\Omega$ as a continuous non-negative real-valued variable, even though it was originally defined as an integer parameter $\Omega \in \mathbb{N}_{+}$. The side bands experiencing the highest gain rate are then obtained from the condition $\zeta+d \Omega^{2}-2 A+\sigma^{\prime} r t_{\mathrm{eff}}^{\prime} \mu A^{2}=0$, which is the only term depending on $\Omega$ in Eq. (12). This leads to

$$
\Omega_{\max }=\sqrt{\left(2 A-\sigma^{\prime} r t_{\mathrm{eff}}^{\prime} \mu A^{2}-\zeta\right) / d}
$$

Note that Eq. (13) implies an appropriate choice of the detuning $\zeta$ such that $\left(2 A-\sigma^{\prime} r t_{\mathrm{eff}}^{\prime} \mu A^{2}-\zeta\right) / d>0$. Note also that a strict derivation of $\Omega_{\max }$ by computing $d \lambda / d \Omega=0$ will yield $\Omega=0$ as an additional local extremum, specifically a local maximum for $d\left(\zeta-2 A+\sigma^{\prime} r t_{\text {eff }}^{\prime} \mu A^{2}\right)>0$ and a local minimum for $d\left(\zeta-2 A+\sigma^{\prime} r t_{\mathrm{eff}}^{\prime} \mu A^{2}\right)<0$. This extremum is not considered further, since the associated gain parameter $\lambda(0)$ is always smaller than $\lambda\left(\Omega_{\max }\right)$, which is given by

$$
\begin{aligned}
\lambda\left(\Omega_{\mathrm{max}}\right) & =-1-r\left(2+\sigma^{\prime} t_{\mathrm{eff}}^{\prime} A\right) A+A \sqrt{r^{2}+1} \\
& =-1+\left(\sqrt{r^{2}+1}-2 r\right) A-\sigma^{\prime} r t_{\mathrm{eff}}^{\prime} A^{2} .
\end{aligned}
$$

Note that Eq. (14) reproduces the well-known fact that $A>1$ is a necessary condition for MI to occur in the absence of TPA and FCA. The presence of these effects may either increase the required normalized power $A$ to values larger than 1 or completely inhibit MI. Specifically, $\lambda\left(\Omega_{\max }\right)$ is negative for any value of $A$ for sufficiently high TPA parameters $r \geqslant 1 / \sqrt{3}$; i.e., MI cannot occur, irrespective of the pump power. On the other hand, positive values of $\lambda\left(\Omega_{\max }\right)$ may be found for certain normalized powers $A$ if both of the following conditions are satisfied:

$$
0 \leqslant r<1 / \sqrt{3}, \quad 0 \leqslant \sigma^{\prime} t_{\mathrm{eff}}^{\prime}<\frac{1}{4 r}\left(\sqrt{r^{2}+1}-2 r\right)^{2} .
$$

Note that the upper limit of $1 / \sqrt{3}$ for the TPA parameter $r$ is exact. In a bifurcation study of the LLE [37], $r=1 / \sqrt{3}$ was also found as a threshold value for the TPA parameter preventing bifurcations from the steady-state solution $\underline{a}\left(t^{\prime}, \tau^{\prime}\right)=\underline{a}_{0}$. Further, $r<1 / \sqrt{3}$ is also a necessary condition for bistability of the continuous-wave (cw) intracavity power $A=\left|\underline{a}_{0}\right|^{2}$ as a function of the detuning $\zeta$ [18]. Note also that other investigations previously described the impact of the TPA parameter on the intracavity power $A$ required for $\mathrm{MI}$ in the presence of TPA and FCA [17] and in the presence of TPA only, see again $[18,19]$. In both cases, the required intracavity (IC) 


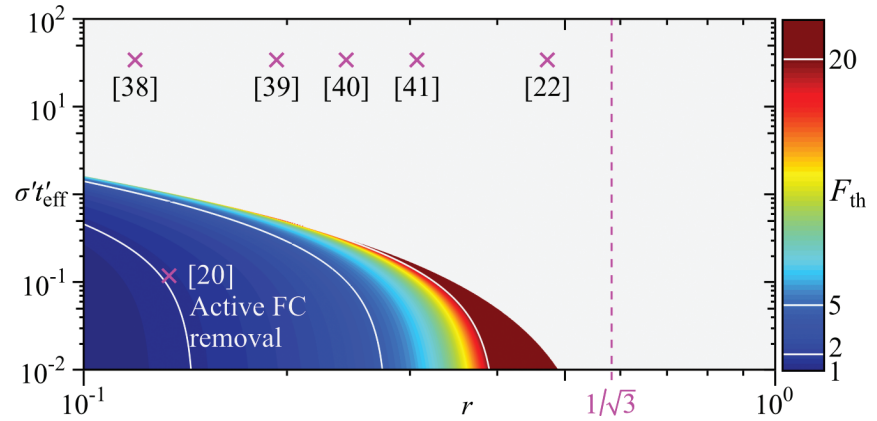

FIG. 2. Threshold forcing needed to achieve MI as a function of $r$ and $\sigma^{\prime} t_{\text {eff }}^{\prime}$. Modulation instability and comb formation can be observed only to the left of the vertical dashed line $r=3^{-1 / 2}$. The gray area indicates the parameter space for which modulation instability does not occur. Magenta crosses mark real data points reported for silicon-photonic waveguides [20,22,38-41], assuming waveguide losses of $\alpha_{i}=46 \mathrm{~m}^{-1}\left(2 \mathrm{~dB} \mathrm{~cm}^{-1}\right)$ [20]. Reference [20] reports on silicon-photonic waveguides in which free carriers are actively removed by a reverse-biased $p-i$ - $n$-junction, leading to a dwell time of $t_{\text {eff }}^{\prime}=12 \mathrm{ps}$. In all other cases, no free-carrier removal was used, leading to dwell times of the order of $1-5 \mathrm{~ns}$ according to [16,20-23]. Specifically, a value of $3 \mathrm{~ns}$ was used for the data points related to Refs. [38-41], which do not specify values for $t_{\text {eff }}^{\prime}$. The value for the FCA cross section $\sigma=1.45 \times 10^{-21} \mathrm{~m}^{2}$ is consistently found in various publications [20,22,39]. The operating wavelength is $1550 \mathrm{~nm}$.

power diverges for $r \rightarrow 1 / \sqrt{3}$. We therefore conclude that a TPA parameter $r \geqslant 1 / \sqrt{3}$ prohibits a multitude of nonlinear phenomena comprising bifurcations from the steady-state solution, bistability, and modulation instability. Also note that the upper limit for the free-carrier lifetime is consistent with the results of [17].

An important quantity to derive is the threshold power that leads to MI in the presence of TPA and FCA. For the values of $r$ and $t_{\text {eff }}^{\prime} \sigma^{\prime}$ specified by Eq. (15), we use Eq. (14) to compute the minimum threshold power $A_{\text {th }}$ of the pumped resonator mode that is required to achieve MI, i.e., $\lambda\left(\Omega_{\max }\right)>0$. The forcing $F_{\text {th }}$ required to achieve $A_{\text {th }}$ is then determined from Eq. (11). For maximizing the power transfer from $F_{\text {th }}$ to $A_{\text {th }}$, the detuning is chosen as $\zeta=A_{\text {th }}+\phi_{0}-\sigma^{\prime} r t_{\text {eff }}^{\prime} \mu A_{\text {th }}^{2}$ by appropriate adjustment of the pump frequency, thus eliminating the expression marked by a star (*) in Eq. (11). For this detuning, maximum gain is found for modes with offset $\Omega_{\max }=\sqrt{\left(A_{\mathrm{th}}-\phi_{0}\right) / d}$. For anomalous dispersion, $d>0$ $\left(\beta_{2}<0\right)$, real-valued $\Omega_{\max }$ can be found as long as $\phi_{0}<A_{\text {th }}$, which includes also the complete absence of AMC, $\phi_{0}=0$. In contrast to that, normal dispersion, $d<0\left(\beta_{2}>0\right)$ requires $\phi_{0}>A_{\mathrm{th}}$, i.e., a spectral shift of the resonance caused by sufficiently strong AMC to yield a real-valued $\Omega_{\max }$. For real $\Omega_{\max }$, Fig. 2 displays the color-coded threshold forcing $F_{\text {th }}$ that is required to achieve MI as a function of the normalized TPA coefficient $r$ and the free-carrier influence $\sigma^{\prime} t_{\text {eff }}^{\prime}$. The color-coded map is limited to the ranges within which MI can be achieved [see Eq. (15)], while the remainder of the plot is kept in gray. We find that $F_{\text {th }}$ increases continuously with increasing $r$ and $\sigma^{\prime} t_{\text {eff }}^{\prime}$, which is caused by both an increase of $A_{\text {th }}$ needed to achieve positive $\lambda\left(\Omega_{\max }\right)$ according to Eq. (14), and a reduced power transfer from the pump $F$ to the pumped mode $A$, Eq. (11). In the absence of FCA, i.e., $\tau_{\text {eff }}^{\prime} \sigma^{\prime}=0$, MI is possible for sufficiently weak TPA $r<1 / \sqrt{3}$, indicated by a vertical dashed line, and the associated threshold forcing $F_{\text {th }}$ diverges for $r \rightarrow 1 / \sqrt{3}$. This can be seen in Eq. (14), wherein the factor $\sqrt{r^{2}+1}-2 r \rightarrow 0$ vanishes for $r \rightarrow 1 / \sqrt{3}$ and thus $A_{\text {th }} \rightarrow \infty$ is needed to achieve positive $\lambda\left(\Omega_{\max }\right)$ for $\sigma^{\prime} t_{\mathrm{eff}}^{\prime} \rightarrow 0$. For $r<1 / \sqrt{3}$ the threshold forcing at the edge of the MI-enabling parameter space remains finite.

Note that all these findings are based on various approximations that make Eq. (10) amenable to an analytic investigation. In Appendix $\mathrm{C}$, we check the result of Fig. 2 for a specific set of parameters $\sigma^{\prime}, \mu, v^{\prime}, d>0$ by evaluating Eqs. (10) and (11) numerically for varying $r$ and $t_{\text {eff }}^{\prime}$ without the approximations involved in Eq. (14). In this investigation, we again assume that AMC is absent, i.e., $\phi_{\Omega^{\prime}}=0 \forall \Omega^{\prime}$. The relative deviation of the threshold forcing found by the numerical evaluation from its analytically approximated counterpart stays below $1 \%$. We hence conclude that the simplified procedure leading to Fig. 2 can be considered sufficiently accurate.

Figure 2 also shows published experimental data (magenta crosses) for the TPA coefficient $r$ and for the product $\sigma^{\prime} t_{\text {eff }}^{\prime}$ of the FC cross section and dwell time. The published values for $r$ are all in the same order of magnitude [20,22,38-41] and stay below the limiting value of $1 / \sqrt{3}$ as given in Eq. (15). For simple silicon-photonic waveguides without active freecarrier removal, dwell times $t_{\text {eff }}^{\prime}$ are of the order of 1-5 ns [16,20-23], thereby clearly inhibiting MI. However, active free-carrier removal by a reverse-biased $p-i$ - $n$-junction can effectively reduce the dwell time to values of, e.g., 12 ps [20], such that modulation instability and frequency comb formation become possible at telecommunication wavelengths. The lower limit for $\sigma^{\prime} t_{\text {eff }}^{\prime}$ that is achievable by active free-carrier removal is dictated by the saturation drift velocity $v_{\mathrm{FC}}$ of the free carriers, which is of the order of $10^{5} \mathrm{~m} \mathrm{~s}^{-1}$ for electrons in silicon [42]. For a microresonator with an assumed waveguide width of $w=480 \mathrm{~nm}$, the theoretically estimated carrier dwell time can be as small as $t_{\mathrm{eff}}=w / v_{\mathrm{FC}}=4.8 \mathrm{ps}$ which leads to $\sigma^{\prime} t_{\text {eff }}^{\prime}=0.05$ and clearly enables MI.

\section{SILICON MICRORESONATOR FOR KERR COMB GENERATION AT TELECOM WAVELENGTHS: DESIGN STUDY AND NUMERICAL SIMUATIONS}

With the general model of Sec. IV at hand, we can now design a specific implementation of a silicon-photonic microresonator which is geared toward Kerr comb generation at telecom wavelengths around $1550 \mathrm{~nm}$. Figure 1 shows the general layout of the device. The silicon waveguide is undoped and is part of the intrinsic zone of a $p$ - $i$ - $n$-junction $[16,28]$. A reverse voltage applied through vertical interconnect accesses (vias) to the $p^{+}$-doped (red) and $n^{-}$-doped (blue) regions of the $p-i-n$-junction leads to efficient removal of free carriers such that dwell times of the order of the round-trip time can be achieved. To determine suitable waveguide parameters, we perform finite-element simulations for a specific waveguide geometry. We choose the width of the center waveguide as $w=480 \mathrm{~nm}$, the height as $260 \mathrm{~nm}$ and the slab height as $40 \mathrm{~nm}$, such that the total height amounts to $h=300 \mathrm{~nm}$. The width of the undoped region is set to 
$1300 \mathrm{~nm}$. This geometry of the cross-sectional design for fast carrier removal is similar to the one used in [20] and results in anomalous group-velocity dispersion around $1550 \mathrm{~nm}$ [29]. Specifically, we obtain a second-order dispersion parameter of $\beta_{2}=-0.587 \mathrm{ps}^{2} \mathrm{~m}^{-1}$, a group refractive index of $n_{\mathrm{g}}=4.15$, and a nonlinearity parameter of $\gamma=257 \mathrm{~W}^{-1} \mathrm{~m}^{-1}$ at $1550 \mathrm{~nm}$; see Appendix D for details. The value of the group refractive index along with a targeted free spectral range (FSR) of $100 \mathrm{GHz}$ leads to a microresonator diameter of 230 $\mu \mathrm{m}$. For the TPA and FCA parameters marked by a magenta cross in Fig. 2 [20], the threshold forcing for MI amounts to $F_{\text {th }}=2.06$. Assuming a power loss coefficient of $\alpha_{i}=46 \mathrm{~m}^{-1}$ $\left(2 \mathrm{~dB} \mathrm{~cm}^{-1}\right)$ and critical coupling, which corresponds to a $Q$ factor of approximately $2 \times 10^{5}$, this threshold forcing translates into a threshold pump power of $P_{\text {in }} \approx 12 \mathrm{~mW}$ measured in the on-chip bus waveguide. $Q$ factors of the order of $10^{5}$ have been demonstrated using commercial silicon-photonic foundry processes [43].

Based on this resonator design, we next perform a time integration of the LLE to validate our theoretical predictions on comb formation and to study the associated impact of fast free-carrier dynamics. Our model accounts for the full dynamics of free carriers both on the slow and on the stationary fast timescale and complements previous investigations of Kerr comb generation in silicon microresonators such as [22], which formulates the free-carrier dynamics on the retarded fast timescale and accounts for the temporal evolution on the slow timescale by appropriate boundary conditions. We start our consideration from the microresonator design described before, which features a normalized group velocity $v^{\prime}=189$, a dispersion coefficient $d=0.0025$, a TPA coefficient $r=$ 0.133 , an FCA cross section $\sigma^{\prime}=2.97$, and an FC dispersion coefficient $\mu=7.5$ [22,44]; see Appendix G, Tables II and III, for a list of the underlying physical microresonator parameters along with their connection to the normalized quantities. Forcing $F$, detuning $\zeta$, and FC dwell time $\tau_{\mathrm{eff}}^{\prime}$ are externally controllable parameters and are varied in our simulations.
We use a discretization of $\Delta \tau^{\prime}=2 \pi / 1024$ ( $\Delta \tau=9.8 \mathrm{fs}$ ) for the normalized fast time by dividing the round-trip time into 1024 parts, and we set the slow-time step size to $\Delta t^{\prime}=64 \times$ $\tau^{\prime} / v^{\prime}=2.1 \times 10^{-3}(\Delta t=64 \times \Delta \tau=626 \mathrm{fs})$. Note that in order to correctly simulate the slow- and fast-time dynamics of both the optical field $\underline{a}\left(t^{\prime}, \tau^{\prime}\right)$ and the free carriers $N_{\mathrm{c}}\left(t^{\prime}, \tau^{\prime}\right)$, the slow-time increment and the fast-time increment are linked; see Appendix E for details. We run each simulation until $t^{\prime}=150$, corresponding to 72174 time steps for the slow time. The initial field for each simulation is given by 1024 complex numbers with random phases between 0 and $2 \pi$ and random amplitudes between 0 and $10^{-14}$. The maximum amplitude of the initial field is chosen such that the power of the initial field is negligible compared to the forcing but can still act as a seed for starting the evolution of the differential equation system. More details on the integration of the coupled Eqs. (5) and (6) can be found in Appendix E. We analyze four different cases, see columns (a-d) of Fig. 3 for the results.

In column (a) (Inhibited modulation instability), the parameters are set as follows: Forcing $F=5$ (pump power $P_{\text {in }}=$ $30 \mathrm{~mW})$, dwell time $t_{\text {eff }}^{\prime}=0.1665\left(t_{\mathrm{eff}}=50 \mathrm{ps}\right)$, detuning $\zeta=2.2(\Delta \omega=2 \pi \times 1.16 \mathrm{GHz})$, and $d=0.0025$, corresponding to anomalous dispersion, i.e., $\beta_{2}<0$; see Table I. The simulation parameters are listed in row R1 of Fig. 3. The dispersion profile of the 101 central frequency comb modes, represented in normalized terms by $\varphi^{\prime}\left(\Omega^{\prime}\right)=-\Omega^{\prime 2} d+\phi_{\Omega^{\prime}}$, is shown in row $\mathrm{R} 2$ as a function of the mode index $\Omega^{\prime}$. In physical terms, the dispersion profile $\varphi\left(\Omega^{\prime}\right)$ corresponds to the phase deviation accumulated by each comb mode $\Omega^{\prime}$ over a single round trip $t_{\mathrm{RT}}$ in the resonator due to dispersion and avoided mode crossings. This can be seen by introducing Eq. (1) into a reduced version of Eq. (2), where only the fifth and sixth term $i\left[-\left(\beta_{2} L / 2\right) \partial^{2} / \partial \tau^{2}+\hat{\Phi}_{\mathrm{AMC}}\right] \underline{E}(t, \tau)$ on the r.h.s. are maintained. Using Eq. (4), and the normalized quantities defined in Table I, we obtain

$$
\begin{aligned}
i\left[-\frac{\beta_{2} L}{2} \frac{\partial^{2}}{\partial \tau^{2}}+\hat{\Phi}_{\mathrm{AMC}}\right] \sum_{\Omega^{\prime}} \underline{E}_{\Omega^{\prime}} e^{i 2 \pi \Omega^{\prime} t / t_{\mathrm{RT}}} & =i \sum_{\Omega^{\prime}} \varphi\left(\Omega^{\prime}\right) \underline{E}_{\Omega^{\prime}} e^{i 2 \pi \Omega^{\prime} t / t_{\mathrm{RT}}}, \\
\varphi\left(\Omega^{\prime}\right) & =\frac{\beta_{2} L}{2}\left(\Omega^{\prime} \times \frac{2 \pi}{t_{\mathrm{RT}}}\right)^{2}+\delta \omega_{\Omega^{\prime}} t_{\mathrm{RT}}, \\
\varphi^{\prime}\left(\Omega^{\prime}\right) & =\varphi\left(\Omega^{\prime}\right) \frac{n_{\mathrm{g}}}{\alpha_{i} t_{\mathrm{RT}} c}=-\Omega^{\prime 2} d+\phi_{\Omega^{\prime}} .
\end{aligned}
$$

The above-mentioned choice of forcing and dwell time ensures that the normalized gain rate $\lambda(\Omega)$ (with $\Omega=\left|\Omega^{\prime}\right|$ ), Eqs. (10) and (11), is always negative; see row R3. As a consequence, modulation instability cannot occur, and the only mode with nonzero power is the pumped mode at modal index $\Omega^{\prime}=0$. In row R4, the color-coded power spectrum $\left|\underline{a}_{\Omega^{\prime}}\left(t^{\prime}\right)\right|^{2}$ is shown as a function of the normalized (slow) time $t^{\prime}$ in the range $0 \leqslant t^{\prime} \leqslant 150$ and of the modal index $\Omega^{\prime}$ in the range $-50 \leqslant \Omega^{\prime} \leqslant+50$. Row R5 displays the final power spectrum at $t^{\prime}=150$ as a function of the modal index $\Omega^{\prime}$. In row R6, the color-coded intracavity (IC) power $\left|\underline{a}\left(t^{\prime}, \tau^{\prime}\right)\right|^{2}$ is depicted as a function of normalized slow time $t^{\prime}$ and normalized fast (retarded) time $\tau^{\prime}$. In the absence of modulation instability, the IC power remains constant along the circumference of the resonator, $\left|a\left(t^{\prime}, \tau^{\prime}\right)\right|^{2}=1.11$; see rows R6 and R7. Similarly, the free-carrier density is also constant, as depicted in row R8.

In column (b) (Modulation instability), we keep all parameters of column (a) except for $t_{\text {eff }}^{\prime}$, which is reduced to 0.0406. This corresponds to a physical dwell time $t_{\text {eff }}=$ $12.2 \mathrm{ps}$, which has previously been demonstrated in a comparable silicon-photonic waveguide [20]. In this case, the computed normalized gain rate is positive within a certain 


\begin{tabular}{|c|c|c|c|c|c|c|c|c|c|c|}
\hline & $\begin{array}{c}\text { In } \\
\text { mo } \\
\text { ins }\end{array}$ & $\begin{array}{l}\text { bite } \\
\text { Ilati } \\
\text { bilit }\end{array}$ & & $\begin{array}{c}\text { Mo } \\
\text { ins }\end{array}$ & $\begin{array}{l}\text { dulation } \\
\text { tability }\end{array}$ & (c) $\underset{\text { Dissip }}{\text { soliton }}$ & $\begin{array}{l}\text { tive Kerr } \\
\text { formation }\end{array}$ & $\begin{array}{l}\text { (d) } \begin{array}{r}\text { Mod } \\
\text { insta } \\
\text { avoided } n\end{array} \\
\end{array}$ & $\begin{array}{l}\text { ulat } \\
\text { bility } \\
\text { lode }\end{array}$ & $\begin{array}{l}\text { on } \\
\text { via } \\
\text { crossing }\end{array}$ \\
\hline R1 & & & & & 2.2 & & 3.03 .8 & & & 2.2 \\
\hline & $\begin{aligned} F & =5 \\
d & =0.0025 \\
v^{\prime} & =189\end{aligned}$ & $\downarrow_{t^{\prime}}$ & $\vec{s}$ & $\begin{array}{l}F=5 \\
d=0.0025 \\
v^{\prime}=189\end{array}$ & $\downarrow_{t^{\prime}}$ & $\begin{aligned} F & =8 \\
d & =0.0025 \\
v^{\prime} & =189\end{aligned}$ & $\begin{array}{l}-90- \\
{ }_{t} \\
120\end{array}$ & $\begin{array}{l}F=5 \\
d=-0.0025 \\
v^{\prime}=189\end{array}$ & $\downarrow t^{\prime}$ & $\vec{\zeta}$ \\
\hline & $\begin{aligned} r & =0.133 \\
\mu & =7.5\end{aligned}$ & & & $\begin{aligned} r & =0.133 \\
\mu & =7.5\end{aligned}$ & $\begin{aligned} \sigma^{\prime} & =2.97 \\
t_{\mathrm{eff}}^{\prime} & =0.0406\end{aligned}$ & $\begin{aligned} r & =0.133 \\
\mu & =7.5\end{aligned}$ & $\begin{aligned} \sigma^{\prime} & =2.97 \\
t_{\mathrm{eff}}^{\prime} & =0.0406\end{aligned}$ & $\begin{aligned} r & =0.133 \\
\mu & =7.5\end{aligned}$ & $t_{\mathrm{eff}}^{\prime}$ & $\begin{array}{l}=2.97 \\
=0.0406\end{array}$ \\
\hline
\end{tabular}
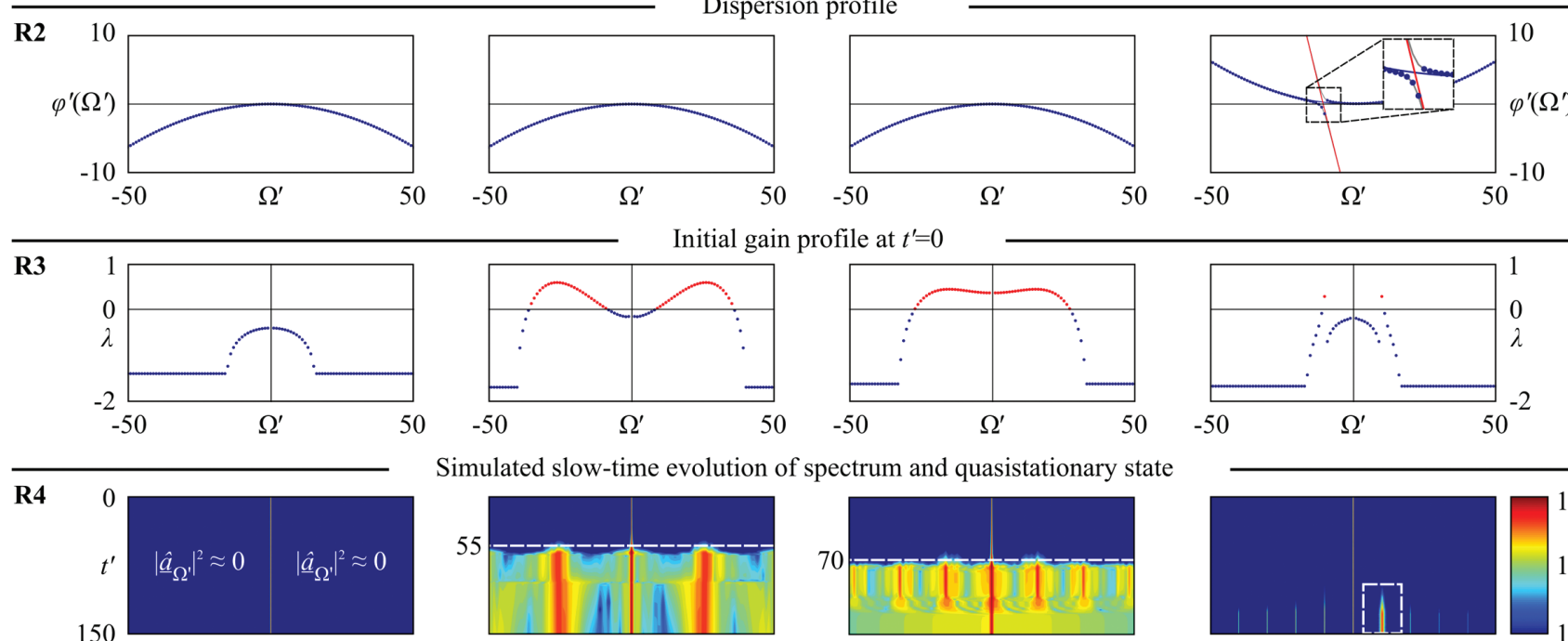

Simulated slow-time evolution of spectrum and quasistationary state
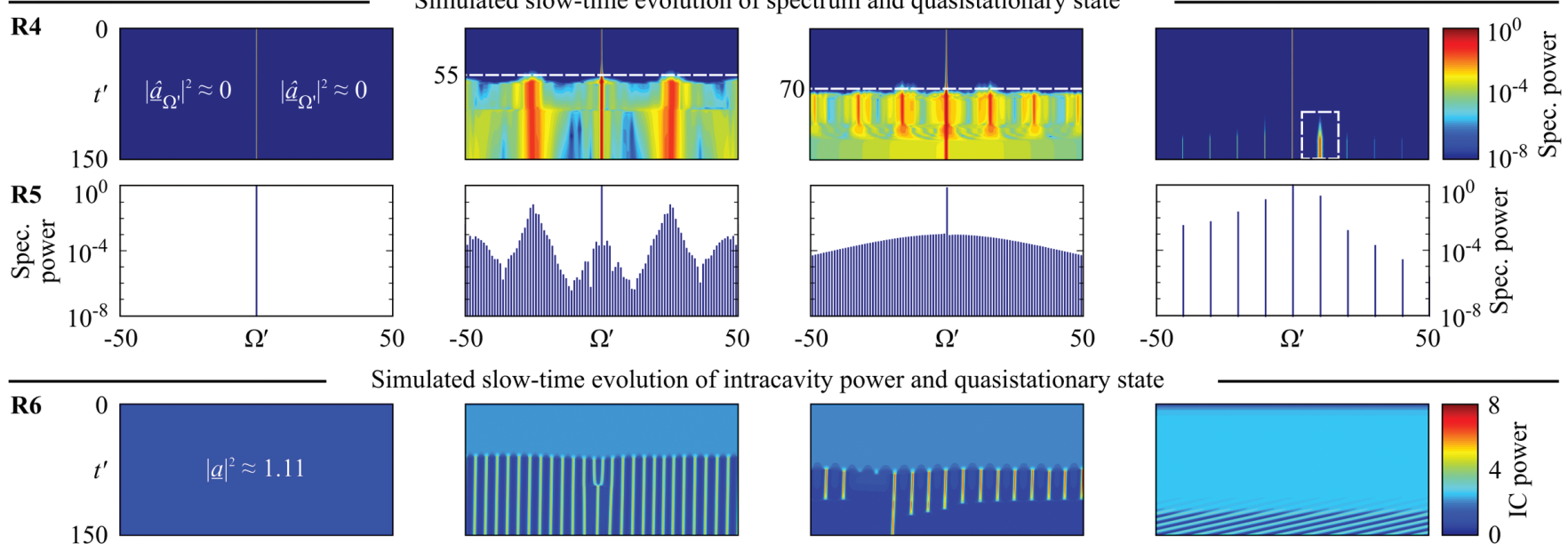

Simulated slow-time evolution of intracavity power and quasistationary state

R7
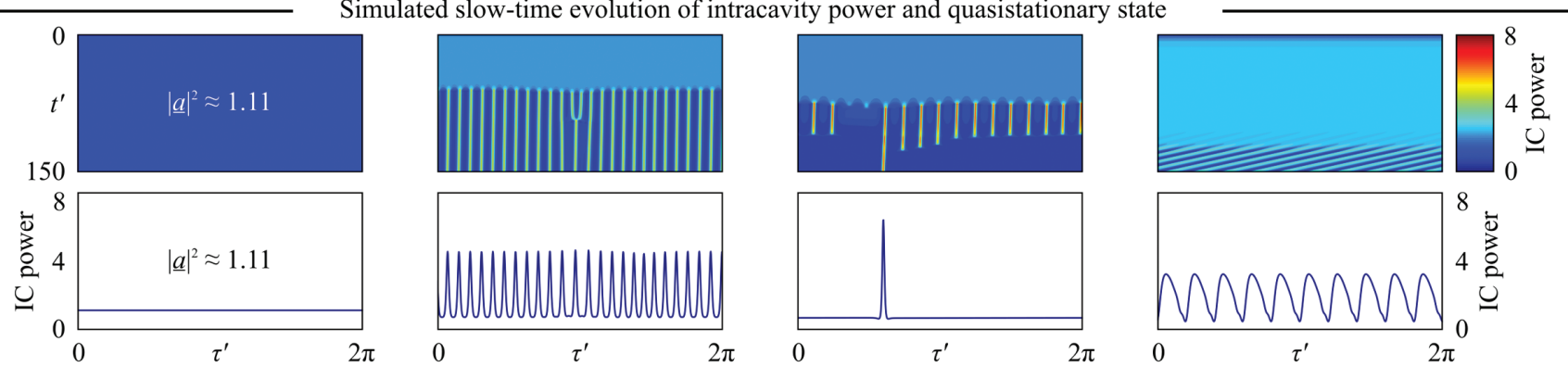

Free-carrier density at final state
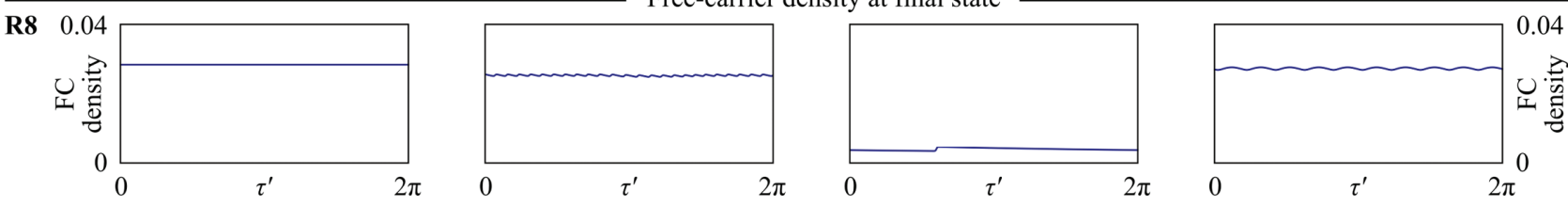

FIG. 3. Results of time integration of the LLE for different resonator designs and pump parameters. Row R1: Simulation parameters. Row R2: Dispersion profile for the central 101 modes. Row R3: Computed normalized gain rates at the beginning of the simulation (slow time $t^{\prime}=0$ ). Row R4: Evolution of power spectra with normalized slow time $t^{\prime}$. Row R5: Spectra at stationary state for $t^{\prime}=150$. Row R6: Color-coded intracavity (IC) power $\left|\underline{a}\left(t^{\prime}, \tau^{\prime}\right)\right|^{2}$ evolving during comb formation over $t^{\prime}$ as a function of the normalized fast time $\tau^{\prime}$. We observe a temporal shift of the IC pulse pattern within the retarded time frame of the fast time axis $\tau^{\prime}$, which can, e.g., be caused by FC dispersion or avoided mode crossings. Besides this shift along the $\tau^{\prime}$ axis, the IC power distribution evolves into a "quasistationary" final state in all cases. Row R7: Quasistationary IC power $\left|\underline{a}\left(t^{\prime}=150, \tau^{\prime}\right)\right|^{2}$. Row R8: Free-carrier density $N_{\mathrm{c}}^{\prime}\left(t^{\prime}=150, \tau^{\prime}-150 v^{\prime}\right)$ at final state. Column $(\mathrm{a})$ : Modulation instability inhibited by long free-carrier dwell time $t_{\mathrm{eff}}$, which corresponds to five times the cavity round-trip time $t_{\mathrm{RT}}$. Column (b): Modulation instability with sufficiently reduced carrier dwell time $t_{\mathrm{eff}}=1.22 \times t_{\mathrm{RT}}$. Column (c): Single-soliton formation for dynamically increasing detuning. Column (d): Comb formation in a normal-dispersion resonator with avoided mode crossing. 
range of modal indices $\Omega^{\prime}$; see Fig. 3(b), row R3 (red dots). During the evolution of the power spectrum with slow time $t^{\prime}$, the first side bands emerge near $t^{\prime}=55$, row R4. A visual comparison with the gain rate plot in row $\mathrm{R} 3$ shows that the positions $\Omega^{\prime} \approx \pm 25$ of the initial side bands coincide with the maxima of the gain parameter $\lambda\left(\Omega^{\prime}\right)$. A power spectrum at $t^{\prime}=150$ is to be seen in row R5. The phase-locked modes lead to a temporal pulse pattern which is visible in rows R6 and R7. Note that there is a small slow drift of this pulse train within the retarded time frame $\tau^{\prime}$, which may be inferred from the nearly vertical, but slightly inclined lines that correspond to the pulse peaks on the fast timescale; see row R6. A possible explanation of this drift might be the fact that the rise of the free-carrier density during each pulse leads to a slightly increased attenuation of the trailing pulse edge and hence to an effectively higher propagation velocity. Mathematically, this behavior can be also seen from the terms $\exp \left( \pm i \lambda_{i} t^{\prime}\right) \exp \left( \pm i \Omega \tau^{\prime}\right)=\exp \left[ \pm i \Omega\left(\lambda_{i} t^{\prime} / \Omega+\tau^{\prime}\right)\right]$ in Eq. (8), which describe a time shift of the optical field $\underline{a}\left(t^{\prime}, \tau^{\prime}\right)$ that continuously increases with slow time $t^{\prime}$ within the fast timescale. The FC density $N_{\mathrm{c}}^{\prime}\left(t^{\prime}, \tau^{\prime}-t^{\prime}\right)$ in row R8 shows modulations. These are, however, small, since the assumed FC lifetime of $12.2 \mathrm{ps}$ is much larger than the narrow temporal spacing of the 25 optical pulses circulating in the cavity, which amounts to approximately $10 \mathrm{ps} / 25=0.4 \mathrm{ps}$.

In column (c) (Dissipative Kerr soliton generation), we increase the forcing to $F=8(48 \mathrm{~mW})$. The detuning is kept constant at $\zeta=3.0$ until $t^{\prime}=90$, then increased linearly to $\zeta=3.8$ until $t^{\prime}=120$, and then kept constant again until the end of the simulation; see row R1 of Fig. 3(c). Such a procedure allows generating single-soliton states [45]. In row $\mathrm{R} 3$, the gain rate is depicted for $t^{\prime}=0$, i.e., before the detuning sweep, showing a broad range of modes that experience parametric gain. In the simulated slow-time evolution of the spectrum, row R4, the first sidebands become visible around $t^{\prime} \approx 70$, and the spectral position of these side bands coincides with the maxima of computed gain rate in row 3 . The maximum of the gain parameter in column (c) is slightly smaller than the one obtained for the scenario described in column (b), and thus the side bands only become visible at a later normalized time $t^{\prime}$. The final power spectrum obtained at the end of the simulation, row R5, is a very regular frequency comb with a smooth envelope, which is typical for a single dissipative Kerr soliton [9] circulating in the ring. The evolution of the color-coded intracavity power, shown in row R6, reveals the emergence of multiple pulses at modulation instability onset around $t^{\prime} \approx 70$. Due to the swept detuning, most of the pulses vanish over time, which is in line with experimental observations [45]. The final value of $\zeta$ was chosen such that a single pulse remains in the cavity, which can be seen from the plot of the IC power $\left|a\left(t^{\prime}=150, \tau^{\prime}\right)\right|^{2}$ in row R7. In this simulation, FCA causes an even more pronounced change of the group velocity as compared to Fig. 3(b), row R6, leading to a stronger temporal shift of the soliton pulse within the retarded time frame $\tau^{\prime}$, while the spectrum remains constant. Such drifts of soliton pulses due to FCA have been reported and investigated in [19]. At higher pump powers, this drift can be even more severe; see, e.g., Appendix E. Comparing the propagation of a single-soliton pulse in the presence of free carriers with and without fast-time dynamics, we find that this drift can only be observed when including these dynamics; see again Appendix E. The free-carrier density, row R8, shows a stronger modulation compared to the previous case shown in column (b). The soliton pulse increases the local free-carrier density by $28 \%$ compared to its cavity mean value. Note that the soliton pulse is accompanied by a constant $\mathrm{cw}$ background, which also contributes a constant part to the FC density. For single-soliton states with stronger pump powers, the fast-time dynamics of the free-carrier density are more pronounced; see again Appendix E for an example. Overall, the FC density is lower compared to cases (a) and (b). This is explained by a reduced FC lifetime compared to (a) and to a significantly reduced number of pulses propagating in the cavity compared to (b).

In column (d) (Modulation instability via avoided mode crossing), the forcing is set back to $F=5$, and the anomalous dispersion profile used in Figs. 3(a)-3(c) is inverted to obtain normal group-velocity dispersion, $d=-0.0025$, see row R1 for all parameters. Additionally, we introduce an avoided mode crossing (AMC) which causes phase shifts $\phi_{\Omega^{\prime}}$ that disturb the dispersion profile. The AMC arises due to a coupling of resonator modes with similar resonance frequency, but different transverse field distributions. The two coupled modes belong to different mode families, characterized by their respective free spectral range. Representing the dispersion profile of the second waveguide mode family in the dispersion diagram of the first mode family leads to equidistant points on an approximately straight line, which is indicated in red in row R2 of column (d). The resonance frequencies of the coupled modes are indicated by blue filled circles connected by gray lines, which deviate from the resonance frequencies of the unperturbed modes $\omega_{\Omega^{\prime}}$ by $\delta \omega_{\Omega^{\prime}}$; see Appendix F and Ref. [46] for details. The FSR of the second mode family and the coupling strength of the two transverse modes are chosen such that the strongest resonance shift amounts to $\delta \omega_{-10}=-2 \pi \times$ $875 \mathrm{MHz}\left(\phi_{-10}=-1.65, \phi_{0}=0.06, \phi_{+10}=0.03\right)$. This corresponds to a local $1 \%$ change of the FSR, which is the same order of magnitude as reported for experimentally investigated microresonators $[33,46]$. The phase shifts $\phi_{\Omega^{\prime}}$ induced by the avoided mode crossing alter the gain parameter such that it becomes positive for certain side bands, with a gain maximum at side bands $\Omega^{\prime}= \pm 10$; see row R3. In the evolution of the power spectrum, row R4, comb lines emerge at these positions. For better visibility, the comb line at $\Omega^{\prime}=10$ is framed by a broken line and horizontally enlarged. Note that pumping a resonance directly adjacent to an avoided mode crossing may allow for generation of dark soliton frequency combs, where the spacing of the comb lines is equal to a single FSR of the microresonator instead of a multiple thereof [47]. Dark solitons might even experience stronger impact from fast free-carrier dynamics than their bright counterparts.

The stationary power spectrum in row R5 shows a pronounced asymmetry. Compared to the previously considered scenarios, the IC power distribution exhibits a stronger temporal shift of the soliton pulse within the retarded time frame $\tau^{\prime}$. This is caused by a contribution of the AMC-induced phase shifts to the imaginary part of the gain rate; see Eq. (10). Apart from this time shift, the final power distribution inside the resonator exhibits a stationary regular pattern, row R7. The FC density is again nearly constant due to the presence of multiple pulses circulating in the cavity; see row R8. 
Note that silicon-photonic waveguides with anomalous group-velocity dispersion around $1550 \mathrm{~nm}$ need a careful design and can only be achieved in a limited parameter space of widths $w$ and heights $h$ [29]. To ensure the technical relevance of the scenarios investigated in columns $(\mathrm{a}-\mathrm{c})$ of Fig. 3, we derived the normalized dispersion parameter along with the corresponding nonlinearity parameter from a specific waveguide design ( $w=480 \mathrm{~nm}, h=300 \mathrm{~nm}$ ); see Table I and Appendix D for details. In contrast to that, normal dispersion can be achieved for a rather large parameter range of waveguide widths and heights, including waveguides with standard heights of $220 \mathrm{~nm}$ or with rather large cross sections that support multimode propagation and avoided mode crossings [29]. Silicon-photonic microresonators corresponding to the scenario considered in column (d) may therefore by realized for multiple different waveguide geometries.

\section{DISCUSSION AND CONCLUSIONS}

The simulation results indicate that modulation instability in silicon-photonic microresonators at telecommunication wavelengths is most likely to be observed if the waveguide is designed for anomalous group-velocity dispersion, if FCA is mitigated by a reverse-biased $p$-i-n-junction which leads to a sufficiently small carrier dwell time, and if the pump power and detuning are chosen properly. If an avoided mode crossing induces local dispersion shifts, microresonators with otherwise normal dispersion can also exhibit modulation instability. In both cases, moderate on-chip pump powers in the range of $10-50 \mathrm{~mW}$ are sufficient to initiate modulation instability and to generate frequency combs. The required pump powers depend strongly on the actual values of the TPA coefficient $\beta_{\text {TPA }}$, which is expressed by the normalized TPA parameter $r$, and on the actual FC dwell time $t_{\text {eff }}$ and its normalized counterpart $t_{\text {eff }}^{\prime}$. We illustrate this dependence in Fig. 4(a), where the on-chip threshold pump power $P_{\text {th }}$ for modulation instability is displayed as a function of the TPA coefficient with the FC dwell time as a parameter. We assume critical coupling, a resonator design, and waveguide properties as described in Sec. V, a Kerr coefficient of $n_{2}=$ $6.5 \times 10^{-18} \mathrm{~m}^{2} \mathrm{~W}^{-1}$, and a pump wavelength of $1550 \mathrm{~nm}$. We find that $t_{\text {eff }}$ has to be of the order of $100 \mathrm{ps}$ or less to enable comb formation across the range of reported values for $\beta_{\text {TPA }}$ in silicon, which reach from 0.45 [38] to $1.5 \mathrm{~cm} \mathrm{GW}^{-1}$ [22] as indicated by vertical dashed lines in Fig. 4(a). For larger dwell times, FCA prevents comb formation, which is indicated by the fact that the green and magenta lines in Fig. 4(a) do not enter the range of reported values for $\beta_{\text {TPA }}$. For $t_{\text {eff }}=10 \mathrm{ps}$ (red), the threshold pump power varies between 10 and $60 \mathrm{~mW}$, close to the threshold powers in the absence of FCA ( $\tau_{\mathrm{eff}}=0 \mathrm{ps}$, black curve) within the range of reported values of $\beta_{\text {TPA }}$. Note that these values for the threshold pump power only apply for an optimized detuning $\zeta$, that minimizes the expression $(*)$ in Eq. (11). Note also that the proper choice pump power and the corresponding optimum detuning $\zeta$ is an essential prerequisite for observing MI and subsequent comb formation. For numerical simulations with nonoptimum pump parameters, MI and comb formation may not be observed [22] even though the underlying device should support these processes in consideration of its TPA and FCA parameters.

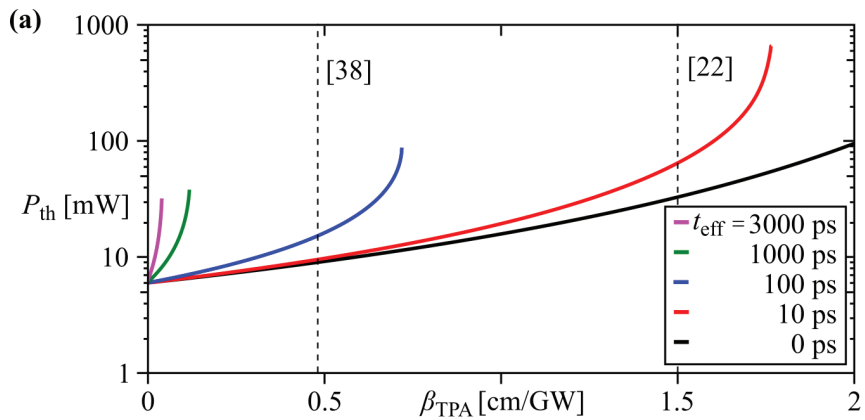

(b)

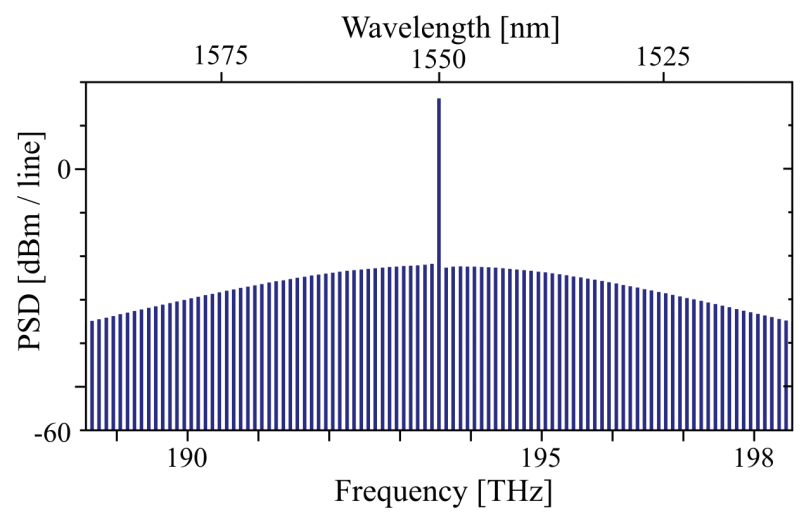

FIG. 4. (a) On-chip threshold pump power for the onset of modulation instability as a function of TPA coefficient $\beta_{\text {TPA }}$ for different FC dwell times $t_{\text {eff }}$. We assume a silicon microresonator with an $f_{\mathrm{FSR}}=100 \mathrm{GHz}$ at $1550 \mathrm{~nm}$; see Sec. V for details. The microresonator is assumed to have $2 \mathrm{~dB} \mathrm{~cm}^{-1}$ waveguide loss, anomalous dispersion, and critical coupling to the bus waveguide. Reported values of $\beta_{\text {TPA }}$ for silicon range from 0.45 to $1.5 \mathrm{~cm} \mathrm{GW}^{-1}$ as indicated by the vertical dashed lines. (b) Soliton comb spectrum obtained in the bus waveguide after the microresonator. The resonator is identical to the one considered in (a). We assume $\beta_{\text {TPA }}=0.7 \mathrm{~cm} \mathrm{GW}^{-1}, t_{\text {eff }}=$ $12 \mathrm{ps}$, and $P=50 \mathrm{~mW}$. The spectrum is derived from the normalized intracavity comb spectrum indicated in Fig. 3(c), row R5.

For illustration of a specific comb source, we again assume a resonator design and waveguide properties as specified in Sec. V, along with a Kerr coefficient of $n_{2}=6.5 \times$ $10^{-18} \mathrm{~m}^{2} \mathrm{~W}^{-1}$ and a pump wavelength of $1550 \mathrm{~nm}$. Based on these parameters, we calculate the physical frequency comb spectrum that is obtained in the bus waveguide after the microresonator; see Fig. 4(b). The TPA coefficient, the effective carrier dwell time, and the pump power are chosen as $0.7 \mathrm{~cm} \mathrm{GW}^{-1}$ [20], $12 \mathrm{ps}$ [20], and $48 \mathrm{~mW}$, respectively. The physical spectrum shown in Fig. 4(b) is derived from the normalized intracavity comb spectrum indicated in Fig. 3(c), row R5.

The model presented in Eqs. (2)-(4) can be further refined by including additional physical effects such as higher-order dispersion, Raman shift [48], or self-steepening. We expect that these effects may lead to minor corrections of the quantitative predictions on modulation instability without changing the qualitative behavior. Note that the incorporation of such additional effects in Eq. (2) is straightforward (see, e.g., [22]), and can be performed independently of our description of stationary free carriers, Eq. (3). Note also that, while the Raman effect has no significant impact on the initial combformation conditions [19], it severely affects the spectrum 
of broadband soliton frequency combs $[19,49,50]$ as well as the power conversion efficiency [51] and the pump parameter space in which solitons can exist [19]. In addition, the results may be improved by using more accurate data for $\beta_{\text {TPA }}$ and $t_{\text {eff }}$, which will help to obtain a more precise estimate of the threshold power for MI-a key parameter from a practical point of view.

For practical devices, further reduction of the pump power is key, especially when it comes to integrated Kerr comb modules in which the available pump power is limited [52-55]. In this context, improvements in waveguide fabrication may allow reducing the linear propagation losses to values of, e.g., $0.4 \mathrm{~dB} \mathrm{~cm}^{-1}$ [56], which is well below the $2 \mathrm{~dB} \mathrm{~cm}^{-1}$ assumed in this work, but still far above the intrinsic absorption of silicon of less than $0.01 \mathrm{~dB} \mathrm{~cm}^{-1}$ at telecommunication wavelengths [57]. As an alternative or an addition to reversebiased $p-i-n$ junctions, silicon self ion implantation may be used to reduce of the $\mathrm{FC}$ dwell time at the expense of slightly increased waveguide losses [58]. Alternative waveguide concepts, e. g., silicon organic hybrid $(\mathrm{SOH})$ waveguides [31,32] with a high Kerr nonlinearity of the order of $\gamma=$ $100 \mathrm{~W}^{-1} \mathrm{~m}^{-1}$ and low normalized TPA absorption coefficients of $r \approx 0.036$, corresponding to $\beta_{\mathrm{TPA}}=0.5 \mathrm{~cm} \mathrm{GW}^{-1}$ for $n_{2}=1.7 \times 10^{-17} \mathrm{~m}^{2} \mathrm{~W}^{-1}$ [31], may also be used in a microresonator.

In summary, we have presented a theoretical analysis of the impact of nonlinear loss mechanisms such as TPA and FCA on Kerr comb formation based on a model that accounts for free-carrier dynamics in a stationary frame for the fast time. Our model is based on normalized quantities and can thus be applied to a wide range of resonator design, materials, and operation conditions, including devices that are affected by avoided mode crossings (AMCs). We derive the maximum two-photon absorption and free-carrier lifetime that still allow for modulation instability at sufficiently low pump powers and that can thus lead to frequency comb formation. We show that microresonators realized on the silicon-photonic platform are not necessarily unsuited for Kerr comb generation at NIR telecommunication wavelengths around 1550 $\mathrm{nm}$, provided that the dwell time of the free carriers in the waveguide core is reduced by a reverse-biased $p-i$ - $n$-junction and that the pump parameters are chosen appropriately, and we present a specific design of a silicon microresonator with anomalous group-velocity dispersion that may even support formation of dissipative Kerr solitons. A numerical solution of the Lugiato-Lefever equation and our suggested free-carrier rate equation shows the onset of comb formation in agreement with our theoretical small-signal analysis. The impact of fasttime free-carrier dynamics is rather small as long as no comb is present or multiple pulses circulate in the microresonator. However, once single-soliton pulses propagate, the FC density clearly deviates from its fast-time mean value, and the optical field shows an additional fast-time drift. Both the numerical and the theoretical small-signal analysis demonstrate that modulation instability can also occur for normal-dispersion resonators if an avoided mode crossing comes into play. This would permit Kerr comb generation in silicon ring resonators with a standard waveguide height of $220 \mathrm{~nm}$. We believe that our findings will help to design microresonators for Kerr comb generation on different photonic integration platforms that are affected by nonlinear loss and free-carrier absorption.

Datasets are available from the corresponding authors upon reasonable request.

\section{ACKNOWLEDGMENTS}

Funded by the Deutsche Forschungsgemeinschaft (DFG, German Research Foundation), Project No. 258734477-SFB 1173 (Collaborative Research Center "WavePhenomena"); European Union H2020 Marie Skłodowska-Curie Actions, Grant No. 812818 (Innovative Training Network "MICROCOMB"); European Research Council (ERC), Grant No. 773248 (ERC Consolidator Grant “TeraSHAPE”); European Union Horizon 2020 Future and Emerging Technologies (FET Open), Grant No. 863322 ("TeraSlice"); Alfried Krupp von Bohlen und Halbach Stiftung (Alfried Krupp von Bohlen und Halbach Foundation).

\section{APPENDIX A: LUGIATO-LEFEVER EQUATION FOR MODELING MODULATION INSTABILITY}

When pumping a nonlinear system with a sinusoidal waveform of constant amplitude, spectral side bands can develop, and the pump amplitude appears modulated. This so-called modulation instability (MI) is the starting point of frequency comb formation in a pumped Kerr-nonlinear optical microresonator. We determine the onset of MI in the presence of two-photon absorption (TPA) and free-carrier absorption (FCA) analytically by a small-signal approximation of the modified Lugiato-Lefever equation (LLE). In the following analysis, we use normalized quantities; see Table I in the main text. The complex optical field amplitude $\underline{E}(t, \tau)$ [Eq. (1) in the main manuscript] is defined with respect to the pump frequency $\omega_{\mathrm{P}}$. It depends on a "slow" time variable $t=m t_{\mathrm{RT}}$ expressed in multiples $m$ of the fixed round-trip time $t_{\mathrm{RT}}=$ $L / v_{\mathrm{g}}$ for a given resonator perimeter $L$ and a group velocity $v_{\mathrm{g}}$ at the pump frequency $\omega_{\mathrm{P}}$, as well as on a "fast" time $\tau=t-z / v_{\mathrm{g}}$, defined by a retarded time frame that depends both on the slow time $t$ and on the local position $z$ inside the resonator. The corresponding normalized optical field according to Table $\mathrm{I}$ is $\underline{a}\left(t^{\prime}, \tau^{\prime}\right)$, where the slow normalized time variable $t^{\prime}$ is assumed to be continuous and where the short normalized time variable $\tau^{\prime}$ defines the position within the cavity in the retarded fast time frame, $\tau^{\prime} \in[0,2 \pi)$. The resonator is driven by a normalized pump field amplitude $\sqrt{F}$. Due to TPA, the optical field generates free carriers (FCs) with a normalized density $N_{\mathrm{c}}^{\prime}$. The free carriers are stationary in the microresonator, and we hence describe them as a function of a stationary (nonretarded) fast time $\tau_{\mathrm{S}}=-z / v_{\mathrm{g}}$, that only depends on the position $z$ within the resonator. Both the optical field and the free-carrier density can be transformed between the stationary and the retarded fast time frame via $\underline{a}\left(t^{\prime}, \tau^{\prime}\right)=\underline{a}\left(t^{\prime}, \tau_{\mathrm{S}}^{\prime}+t^{\prime} v^{\prime}\right)$ and $N_{\mathrm{c}}^{\prime}\left(t^{\prime}, \tau^{\prime}-t^{\prime} v^{\prime}\right)=N_{\mathrm{c}}^{\prime}\left(t^{\prime}, \tau_{\mathrm{S}}^{\prime}\right)$, where $v^{\prime}$ is the normalized group velocity. Each rapidly varying time-harmonic component $e^{i \omega_{\Omega^{\prime}} \tau}, \omega_{\Omega^{\prime}}=\Omega^{\prime} \times\left(2 \pi / t_{\mathrm{RT}}\right)$, $\Omega^{\prime}=0, \pm 1, \pm 2, \pm 3 \cdots$ in Eq. (1) of the main text is periodic in $\tau$ with the round-trip time, leading to periodic boundary conditions $\underline{a}\left(t^{\prime}, \tau^{\prime}\right)=\underline{a}\left(t^{\prime}, \tau^{\prime}+2 \pi\right)$ for the dependence of the complex field amplitude on the normalized fast time $\tau^{\prime}$, 
which can hence be represented as a Fourier series, $\underline{a}\left(t^{\prime}, \tau^{\prime}\right)=$ $\sum_{\Omega^{\prime}} \underline{a}_{\Omega^{\prime}}\left(t^{\prime}\right) e^{i \Omega^{\prime} \tau^{\prime}}$. In the following, we describe the slow-time evolution of the optical field $a\left(t^{\prime}, \tau^{\prime}\right)$ in the retarded time frame $\tau^{\prime}$ using the normalized LLE; see Eq. (5) in the main text. Further, we describe the slow-time evolution of the free carriers $N_{\mathrm{c}}^{\prime}\left(t^{\prime}, \tau_{\mathrm{S}}^{\prime}\right)$ in the stationary time frame $\tau_{\mathrm{S}}^{\prime}$. The normalized LLE and the differential equation describing the evolution of the free-carrier density ("free-carrier equation") are given in Eqs. (5) and (6) in the main text,

$$
\begin{aligned}
& \frac{\partial \underline{a}\left(t^{\prime}, \tau^{\prime}\right)}{\partial t^{\prime}} \\
& =\sqrt{F}+\left[-1-i \zeta+i d \frac{\partial^{2}}{\partial \tau^{\prime 2}}+i \hat{\Phi}_{\mathrm{AMC}}^{\prime}+(i-r)\right. \\
& \left.\quad \times\left|\underline{a}\left(t^{\prime}, \tau^{\prime}\right)\right|^{2}-\sigma^{\prime}(1+i \mu) N_{\mathrm{c}}^{\prime}\left(t^{\prime}, \tau^{\prime}-t^{\prime} v^{\prime}\right)\right] \underline{a}\left(t^{\prime}, \tau^{\prime}\right), \\
& \frac{\partial N_{\mathrm{c}}^{\prime}\left(t^{\prime}, \tau_{\mathrm{S}}^{\prime}\right)}{\partial t^{\prime}}=r\left|\underline{a}\left(t^{\prime}, \tau_{\mathrm{S}}^{\prime}+t^{\prime} v^{\prime}\right)\right|^{4}-\frac{N_{\mathrm{c}}^{\prime}\left(t^{\prime}, \tau_{\mathrm{S}}^{\prime}\right)}{t_{\mathrm{eff}}^{\prime}} .
\end{aligned}
$$

The normalized difference between the pump frequency $\omega_{\mathrm{P}}$ and the closest resonance frequency $\omega_{\mathrm{R}}$ of an unpumped resonator is $\zeta$. The second-order dispersion of the microresonator is represented by the normalized dispersion parameter $d$. The quantity $r$ denotes the normalized TPA coefficient. For the TPA-generated FC, the quantities $\sigma^{\prime}, \mu, t_{\text {eff }}^{\prime}$ define the normalized absorption cross section, the contribution to the refractive index, and the normalized dwell time inside the microresonator waveguide, respectively. The relationship between normalized parameters and physical quantities are summarized in Table I of the main text. We further include an operator $\hat{\Phi}_{\mathrm{AMC}}^{\prime}$ describing phase shifts $\phi_{\Omega^{\prime}}$ experienced by individual components $\underline{a}_{\Omega^{\prime}}\left(t^{\prime}\right)$ of the optical field for frequencies $\Omega^{\prime}$. These phase shifts take into account local resonance frequency shifts caused by mode coupling of different transverse modes, an effect known as avoided mode crossing [46]. The operator can be written as

$$
\begin{aligned}
\hat{\Phi}_{\mathrm{AMC}}^{\prime} \underline{a}\left(t^{\prime}, \tau^{\prime}\right)= & \sum_{\tilde{\Omega}} \frac{\phi_{\tilde{\Omega}}}{2 \pi} \int_{0}^{2 \pi} \underline{a}\left(t^{\prime}, \tau_{1}\right) \\
& \times \exp \left[-i \tilde{\Omega} \tau_{1}\right] d \tau_{1} \exp \left[i \tilde{\Omega} \tau^{\prime}\right] .
\end{aligned}
$$

In our simulations shown in the main text, the strength of the mode coupling, which defines the exact values $\phi_{\Omega^{\prime}}$, was chosen to reflect typical experimental results.

To investigate the condition for the onset of MI, we assume that the optical field consists of a constant pumped mode $\underline{a}_{0}$ and two infinitesimally small side bands $\underline{a}_{ \pm \Omega}(\Omega=1,2,3 \cdots)$ [59], the temporal evolution of which is described by complex gain parameter $\underline{\lambda}=\lambda+i \lambda_{i}$ :

$$
\begin{aligned}
\underline{a}\left(t^{\prime}, \tau^{\prime}\right) & =\underline{a}_{0}+\underline{a}_{+\Omega}+\underline{a}_{-\Omega}, \quad\left|\underline{\hat{a}}_{ \pm \Omega}\right| \ll\left|\underline{a}_{0}\right|, \\
\underline{a}_{+\Omega} & =\underline{\hat{a}}_{+\Omega} e^{\lambda t^{\prime}} e^{i \lambda_{i} t^{\prime}} e^{i \Omega \tau^{\prime}} \\
\underline{a}_{-\Omega} & =\underline{\hat{a}}_{-\Omega} e^{\lambda t^{\prime}} e^{-i \lambda_{i} t^{\prime}} e^{-i \Omega \tau^{\prime}} .
\end{aligned}
$$

In the stationary fast time frame, this optical field is then given by

$$
\begin{aligned}
\underline{a}\left(t^{\prime}, \tau_{\mathrm{S}}^{\prime}+t^{\prime} v^{\prime}\right) & =\underline{a}_{0}+\underline{a}_{+\Omega, \mathrm{S}}+\underline{a}_{-\Omega, \mathrm{S}}, \\
\underline{a}_{+\Omega, \mathrm{S}} & =\underline{\hat{a}}_{+\Omega} e^{\lambda t^{\prime}} e^{+i \lambda_{i} t^{\prime}} e^{+i \Omega \tau_{\mathrm{S}}^{\prime}} e^{+i \Omega t^{\prime} v^{\prime}} \\
\underline{a}_{-\Omega, \mathrm{S}} & =\underline{\hat{a}}_{-\Omega} e^{\lambda t^{\prime}} e^{-i \lambda_{i} t^{\prime}} e^{-i \Omega \tau_{\mathrm{S}}^{\prime}} e^{-i \Omega t^{\prime} v^{\prime}} .
\end{aligned}
$$

We further assume that right at the onset of MI at $t^{\prime}=0$, the normalized free-carrier density according to Eq. (A2) has reached a stationary value of $N_{\mathrm{c}}^{\prime}\left(0, \tau_{\mathrm{S}}^{\prime}\right)=r t_{\mathrm{eff}}^{\prime}\left|\underline{a}_{0}\right|^{4}$ dictated by the strong pump $\underline{a}_{0}$. Solving Eq. (A2) with this initial condition leads to

$$
N_{\mathrm{c}}^{\prime}\left(t^{\prime}, \tau_{\mathrm{S}}^{\prime}\right)=\exp \left[-\frac{t^{\prime}}{t_{\mathrm{eff}}^{\prime}}\right]\left[r t_{\mathrm{eff}}^{\prime}\left|\underline{a}_{0}\right|^{4}+r \int_{0}^{t^{\prime}}\left|\underline{a}\left(t_{1}, \tau_{\mathrm{S}}^{\prime}+t_{1} v^{\prime}\right)\right|^{4} \exp \left(\frac{t_{1}}{t_{\mathrm{eff}}^{\prime}}\right) d t_{1}\right] .
$$

We then insert Eq. (A5) into Eq. (A6) and apply a small-signal approximation of the form $\left|\underline{a}\left(t^{\prime}, \tau_{\mathrm{S}}^{\prime}+t^{\prime} v^{\prime}\right)\right|^{4} \approx\left|\underline{a}_{0}\right|^{4}+$ $2\left|\underline{a}_{0}\right|^{2}\left(\underline{a}_{0}^{*} \underline{a}_{+\Omega, \mathrm{S}}+\underline{a}_{0} \underline{a}_{-\Omega, \mathrm{S}}^{*}\right)+2\left|\underline{a}_{0}\right|^{2}\left(\underline{a}_{0}^{*} \underline{a}_{-\Omega, \mathrm{S}}+\underline{a}_{0} \underline{a}_{+\Omega, \mathrm{S}}^{*}\right)$, where the star $\left(^{*}\right)$ denotes the complex conjugate. Keeping only terms up to linear order in $\underline{\hat{a}}_{ \pm \Omega}$ or $\underline{\hat{a}}_{ \pm \Omega}^{*}$, we solve the integral in Eq. (A6) and obtain

$$
\begin{aligned}
N_{\mathrm{c}}^{\prime}\left(t^{\prime}, \tau_{\mathrm{S}}^{\prime}\right)= & r t_{\mathrm{eff}}^{\prime}\left|\underline{a}_{0}\right|^{4}+r t_{\mathrm{eff}}^{\prime} 2\left|\underline{a}_{0}\right|^{2}\left(\underline{a}_{0}^{*} \underline{\hat{a}}_{+\Omega}+\underline{a}_{0} \underline{\hat{a}}_{-\Omega}^{*}\right) e^{+i \Omega \tau_{\mathrm{S}}^{\prime}} \frac{1}{t_{\mathrm{eff}}^{\prime} \lambda+1+i t_{\mathrm{eff}}^{\prime}\left(\lambda_{i}+\Omega v^{\prime}\right)}\left(e^{\lambda t^{\prime}} e^{+i\left(\lambda_{i}+\Omega v^{\prime}\right) t^{\prime}}-e^{-t^{\prime} / t_{\mathrm{eff}}^{\prime}}\right) \\
& +r t_{\mathrm{eff}}^{\prime} 2\left|\underline{a}_{0}\right|^{2}\left(\underline{a}_{0}^{*} \underline{\hat{a}}_{-\Omega}+\underline{a}_{0} \underline{\hat{a}}_{+\Omega}^{*}\right) e^{-i \Omega \tau_{\mathrm{S}}^{\prime}} \frac{1}{t_{\mathrm{eff}}^{\prime} \lambda+1-i t_{\mathrm{eff}}^{\prime}\left(\lambda_{i}+\Omega v^{\prime}\right)}\left(e^{\lambda t^{\prime}} e^{-i\left(\lambda_{i}+\Omega v^{\prime}\right) t^{\prime}}-e^{-t^{\prime} / t_{\mathrm{eff}}^{\prime}}\right) .
\end{aligned}
$$

Over time, exponentially decaying terms $e^{-t^{\prime} / t_{\text {eff }}^{\prime}}$ can be neglected since normalized free-carrier lifetimes are of the order of $t_{\mathrm{eff}}^{\prime} \approx 0.1-1$ (corresponding to physical FC lifetimes of the order of $100 \mathrm{ps}$ or less for our microresonator design) such that the decay of $e^{-t^{\prime} / t_{\text {eff }}^{\prime}}$ occurs much faster than the increase of $e^{\lambda t^{\prime}}$ with typical values of $\lambda \approx 0.1$ for the gain parameter. Similarly, for short normalized free-carrier lifetimes $t_{\mathrm{eff}}^{\prime} \approx 0.1-1$ and small gain parameters $\lambda \approx 0.1$, we may approximate $t_{\mathrm{eff}}^{\prime} \lambda+1 \approx 1$ in the denominators in Eq. (A7). In the same manner, we may approximate $\lambda_{i}+\Omega v^{\prime} \approx \Omega v^{\prime}$ in the denominators of Eq. (A7). This is justified by the fact that $\lambda_{i}$ represents the normalized frequency offset of the MI side band from the center of the corresponding resonance and is thus much smaller than the offset from the pump, which corresponds to multiple FSRs and is represented by $\Omega v^{\prime}$, i.e., $\lambda_{i} \ll \Omega v^{\prime}$. The remaining expression for the free-carrier density reads

$$
N_{\mathrm{c}}^{\prime}\left(t^{\prime}, \tau_{\mathrm{S}}^{\prime}\right)=r t_{\mathrm{eff}}^{\prime}\left|\underline{a}_{0}\right|^{2}\left[\left|\underline{a}_{0}\right|^{2}+2 \frac{1-i \Omega t_{\mathrm{eff}}^{\prime} v^{\prime}}{1+\left(\Omega t_{\mathrm{eff}}^{\prime} v^{\prime}\right)^{2}}\left(\underline{a}_{0}^{*} \underline{a}_{+\Omega, \mathrm{S}}+\underline{a}_{0} \underline{a}_{-\Omega, \mathrm{S}}^{*}\right)+2 \frac{1+i \Omega t_{\mathrm{eff}}^{\prime} v^{\prime}}{1+\left(\Omega t_{\mathrm{eff}}^{\prime} v^{\prime}\right)^{2}}\left(\underline{a}_{0}^{*} \underline{a}_{-\Omega, \mathrm{S}}+\underline{a}_{0} \underline{a}_{+\Omega, \mathrm{S}}^{*}\right)\right] .
$$

This result is now transformed into the retarded time frame via $N_{\mathrm{c}}^{\prime}\left(t^{\prime}, \tau^{\prime}-t^{\prime} v^{\prime}\right)=N_{\mathrm{c}}^{\prime}\left(t^{\prime}, \tau_{\mathrm{S}}^{\prime}\right)$, which yields

$$
N_{\mathrm{c}}^{\prime}\left(t^{\prime}, \tau^{\prime}-t^{\prime} v^{\prime}\right)=r t_{\mathrm{eff}}^{\prime}\left|\underline{a}_{0}\right|^{2}\left[\left|\underline{a}_{0}\right|^{2}+2 \frac{1-i \Omega t_{\mathrm{eff}}^{\prime} v^{\prime}}{1+\left(\Omega t_{\mathrm{eff}}^{\prime} v^{\prime}\right)^{2}}\left(\underline{a}_{0}^{*} \underline{a}_{+\Omega}+\underline{a}_{0} \underline{a}_{-\Omega}^{*}\right)+2 \frac{1+i \Omega t_{\mathrm{eff}}^{\prime} v^{\prime}}{1+\left(\Omega t_{\mathrm{eff}}^{\prime} v^{\prime}\right)^{2}}\left(\underline{a}_{0}^{*} \underline{a}_{-\Omega}+\underline{a}_{0} \underline{a}_{+\Omega}^{*}\right)\right] .
$$


The expression for the free-carrier density in the retarded fast time frame is substituted in Eq. (A1) along with the ansatz for $\underline{a}\left(t^{\prime}, \tau^{\prime}\right)$, Eq. (A4). Again, we keep only terms up to linear order in $\underline{\hat{a}}_{ \pm \Omega}$ or $\underline{\hat{a}}_{ \pm \Omega}^{*}$. We separate nonoscillating terms from terms oscillating with $e^{i \Omega \tau^{\prime}}$ and $e^{-i \Omega \tau^{\prime}}$ and compare the associated coefficients. This leads to

$$
\begin{aligned}
& 0=\sqrt{F}+\left[-1-i \zeta+i \phi_{0}+(i-r)\left|\underline{a}_{0}\right|^{2}-\sigma^{\prime}(1+i \mu) r t_{\mathrm{eff}}^{\prime}\left|\underline{a}_{0}\right|^{4}\right] \underline{a}_{0}, \\
& \underline{\lambda}_{+\Omega}= {\left[-1-i \zeta-i d \Omega^{2}+i \phi_{+\Omega}\right] \underline{a}_{+\Omega}+(i-r)\left[2\left|\underline{a}_{0}\right|^{2} \underline{a}_{+\Omega}+\left(\underline{a}_{0}\right)^{2} \underline{a}_{-\Omega}^{*}\right] } \\
&-(1+i \mu) \frac{\sigma^{\prime} r t_{\mathrm{eff}}^{\prime}\left|\underline{a}_{0}\right|^{2}}{1+\left(\Omega t_{\mathrm{eff}}^{\prime} v^{\prime}\right)^{2}}\left[\left(3+\left(\Omega t_{\mathrm{eff}}^{\prime} v^{\prime}\right)^{2}\right)\left|\underline{a}_{0}\right|^{2} \underline{a}_{+\Omega}+2\left(\underline{a}_{0}\right)^{2} \underline{a}_{-\Omega}^{*}-i 2 \Omega t_{\mathrm{eff}}^{\prime} v^{\prime}\left(\left|\underline{a}_{0}\right|^{2} \underline{a}_{+\Omega}+\left(\underline{a}_{0}\right)^{2} \underline{a}_{-\Omega}^{*}\right)\right], \\
& \underline{\lambda}^{*} \underline{a}_{-\Omega}= {\left[-1-i \zeta-i d \Omega^{2}+i \phi_{-\Omega}\right] \underline{a}_{-\Omega}+(i-r)\left[2\left|\underline{a}_{0}\right|^{2} \underline{a}_{-\Omega}+\left(\underline{a}_{0}\right)^{2} \underline{a}_{+\Omega}^{*}\right] } \\
&-(1+i \mu) \frac{\sigma^{\prime} r t_{\mathrm{eff}}^{\prime}\left|\underline{a}_{0}\right|^{2}}{1+\left(\Omega t_{\mathrm{eff}}^{\prime} v^{\prime}\right)^{2}}\left[\left(3+\left(\Omega t_{\mathrm{eff}}^{\prime} v^{\prime}\right)^{2}\right)\left|\underline{a}_{0}\right|^{2} \underline{a}_{-\Omega}+2\left(\underline{a}_{0}\right)^{2} \underline{a}_{+\Omega}^{*}+i 2 \Omega t_{\mathrm{eff}}^{\prime} v^{\prime}\left(\left|\underline{a}_{0}\right|^{2} \underline{a}_{-\Omega}+\left(\underline{a}_{0}\right)^{2} \underline{a}_{+\Omega}^{*}\right)\right] .
\end{aligned}
$$

We divide Eq. (A11) by $e^{\lambda t^{\prime}} e^{i \lambda_{i} t^{\prime}} e^{i \Omega \tau^{\prime}}$ and Eq. (A12) by $e^{\lambda t^{\prime}} e^{-i \lambda_{i} t^{\prime}} e^{-i \Omega \tau^{\prime}}$, and we introduce $\bar{\phi}=\left(\phi_{+\Omega}+\phi_{-\Omega}\right) / 2$ and $\Delta \phi=$ $\left(\phi_{+\Omega}-\phi_{-\Omega}\right) / 2$. Both equations are then expressed in terms of $\underline{\hat{a}}_{ \pm \Omega}$ instead of in terms of $\underline{a}_{ \pm \Omega}$,

$$
\begin{aligned}
\underline{\hat{a}}_{+\Omega}= & {\left[-1-i \zeta-i d \Omega^{2}+i \bar{\phi}+i \Delta \phi\right] \underline{\hat{a}}_{+\Omega}+(i-r)\left[2\left|\underline{a}_{0}\right|^{2} \underline{\hat{a}}_{+\Omega}+\left(\underline{a}_{0}\right)^{2} \underline{\hat{a}}_{-\Omega}^{*}\right] } \\
& -(1+i \mu) \frac{\sigma^{\prime} r t_{\mathrm{eff}}^{\prime}\left|\underline{a}_{0}\right|^{2}}{1+\left(\Omega t_{\mathrm{eff}}^{\prime} v^{\prime}\right)^{2}}\left\{\left[3+\left(\Omega t_{\mathrm{eff}}^{\prime} v^{\prime}\right)^{2}\right]\left|\underline{a}_{0}\right|^{2} \underline{\hat{a}}_{+\Omega}+2\left(\underline{a}_{0}\right)^{2} \underline{\hat{a}}_{-\Omega}^{*}-i 2 \Omega t_{\mathrm{eff}}^{\prime} v^{\prime}\left[\left|\underline{a}_{0}\right|^{2} \underline{\hat{a}}_{+\Omega}+\left(\underline{a}_{0}\right)^{2} \underline{\hat{a}}_{-\Omega}^{*}\right]\right\}, \\
\underline{\lambda}^{*} \underline{\hat{a}}_{-\Omega}= & {\left[-1-i \zeta-i d \Omega^{2}+i \bar{\phi}-i \Delta \phi\right] \underline{\hat{a}}_{-\Omega}+(i-r)\left[2\left|\underline{a}_{0}\right|^{2} \underline{\hat{a}}_{-\Omega}+\left(\underline{a}_{0}\right)^{2} \underline{\hat{a}}_{+\Omega}^{*}\right] } \\
& -(1+i \mu) \frac{\sigma^{\prime} r t_{\mathrm{eff}}^{\prime}\left|\underline{a}_{0}\right|^{2}}{1+\left(\Omega t_{\mathrm{eff}}^{\prime} v^{\prime}\right)^{2}}\left\{\left[3+\left(\Omega t_{\mathrm{eff}}^{\prime} v^{\prime}\right)^{2}\right]\left|\underline{a}_{0}\right|^{2} \underline{\hat{a}}_{-\Omega}+2\left(\underline{a}_{0}\right)^{2} \hat{\underline{a}}_{+\Omega}^{*}+i 2 \Omega t_{\mathrm{eff}}^{\prime} v^{\prime}\left[\left|\underline{a}_{0}\right|^{2} \underline{a}_{-\Omega}+\left(\underline{a}_{0}\right)^{2} \hat{a}_{+\Omega}^{*}\right]\right\} .
\end{aligned}
$$

We subtract $i \Delta \phi \underline{\hat{a}}_{+\Omega}$ in Eq. (A13), add $i \Delta \phi \underline{\hat{a}}_{-\Omega}$ in Eq. (A14), and introduce $\underline{\lambda}^{\prime}=\lambda+i \lambda_{i}^{\prime}=\lambda+i\left(\lambda_{i}-\Delta \phi\right)$. Then, we compute the complex conjugate of the resulting Eq. (A14). We obtain a matrix equation of the form

$$
\begin{aligned}
\underline{\lambda}^{\prime}\left(\frac{\hat{a}_{+\Omega}}{\underline{\hat{a}}_{-\Omega}^{*}}\right)= & \left(\begin{array}{cc}
-1-i \zeta-i d \Omega^{2}+i \bar{\phi}+(i-r) 2\left|\underline{a}_{0}\right|^{2} & (i-r)\left(\underline{a}_{0}\right)^{2} \\
(-i-r)\left(\underline{a}_{0}^{*}\right)^{2} & -1+i \zeta+i d \Omega^{2}-i \bar{\phi}+(-i-r) 2\left|\underline{a}_{0}\right|^{2}
\end{array}\right)\left(\begin{array}{c}
\hat{a}_{+\Omega} \\
\underline{\hat{a}}_{-\Omega}^{*}
\end{array}\right) \\
& -\frac{\sigma^{\prime} r t_{\mathrm{eff}}^{\prime}\left|\underline{a}_{0}\right|^{2}}{1+\left(\Omega t_{\mathrm{eff}}^{\prime} v^{\prime}\right)^{2}}\left(\begin{array}{cc}
(1+i \mu)\left[3+\left(\Omega t_{\mathrm{eff}}^{\prime} v^{\prime}\right)^{2}\right]\left|\underline{a}_{0}\right|^{2} & (1+i \mu) 2\left(\underline{a}_{0}\right)^{2} \\
(1-i \mu) 2\left(\underline{a}_{0}^{*}\right)^{2} & (1-i \mu)\left(3+\left(\Omega t_{\mathrm{eff}}^{\prime} v^{\prime}\right)^{2}\right)\left|\underline{a}_{0}\right|^{2}
\end{array}\right)\left(\begin{array}{c}
\hat{a}_{+\Omega} \\
\underline{\hat{a}}_{-\Omega}^{*}
\end{array}\right) \\
& +i \frac{\sigma^{\prime} r t_{\mathrm{eff}}^{\prime}\left|\underline{a}_{0}\right|^{2}}{1+\left(\Omega t_{\mathrm{eff}}^{\prime} v^{\prime}\right)^{2}} 2 \Omega t_{\mathrm{eff}}^{\prime} v^{\prime}\left(\begin{array}{cc}
(1+i \mu)\left|\underline{a}_{0}\right|^{2} & (1+i \mu)\left(\underline{a}_{0}\right)^{2} \\
(1-i \mu)\left(\underline{a}_{0}^{*}\right)^{2} & (1-i \mu)\left|\underline{a}_{0}\right|^{2}
\end{array}\right)\left(\begin{array}{c}
\underline{\hat{a}}_{+\Omega} \\
\underline{\hat{a}}_{-\Omega}^{*}
\end{array}\right) .
\end{aligned}
$$

This equation can be represented as

$$
\begin{aligned}
\underline{\lambda}^{\prime}\left(\begin{array}{c}
\hat{a}_{+\Omega} \\
\underline{\hat{a}}_{-\Omega}^{*}
\end{array}\right) & =\left(\begin{array}{ll}
\underline{m}_{A, 1}+i \underline{m}_{B, 1} & \underline{m}_{A, 2}+i \underline{m}_{B, 2} \\
\underline{m}_{A, 2}^{*}+i \underline{m}_{B, 2}^{*} & \underline{m}_{A, 1}^{*}+i \underline{\underline{m}}_{B, 1}^{*}
\end{array}\right)\left(\begin{array}{c}
\hat{a}_{+\Omega} \\
\underline{\hat{a}}_{-\Omega}^{*}
\end{array}\right)=\mathbf{M}\left(\begin{array}{l}
\hat{a}_{+\Omega} \\
\underline{\hat{a}}_{-\Omega}^{*}
\end{array}\right), \text { where } \\
\underline{m}_{A, 1} & =-1-i \zeta-i d \Omega^{2}+i \bar{\phi}+(i-r) 2\left|\underline{a}_{0}\right|^{2}-\frac{\sigma^{\prime} t_{\mathrm{eff}}^{\prime}\left|\underline{a}_{0}\right|^{2}}{1+\left(\Omega t_{\mathrm{eff}}^{\prime} v^{\prime}\right)^{2}}(1+i \mu)\left[3+\left(\Omega t_{\mathrm{eff}}^{\prime} v^{\prime}\right)^{2}\right]\left|\underline{a}_{0}\right|^{2}, \\
\underline{m}_{A, 2} & =(i-r)\left(\underline{a}_{0}\right)^{2}-\frac{\sigma^{\prime} r t_{\mathrm{eff}}^{\prime}\left|\underline{a}_{0}\right|^{2}}{1+\left(\Omega t_{\mathrm{eff}}^{\prime} v^{\prime}\right)^{2}}(1+i \mu) 2\left(\underline{a}_{0}\right)^{2}, \\
\underline{m}_{B, 1} & =\frac{\sigma^{\prime} r t_{\mathrm{eff}}^{\prime}\left|\underline{a}_{0}\right|^{2}}{1+\left(\Omega t_{\mathrm{eff}}^{\prime} v^{\prime}\right)^{2}} 2 \Omega t_{\mathrm{eff}}^{\prime} v^{\prime}(1+i \mu)\left|\underline{a}_{0}\right|^{2}, \\
\underline{m}_{B, 2} & =\frac{\sigma^{\prime} r t_{\mathrm{eff}}^{\prime}\left|\underline{a}_{0}\right|^{2}}{1+\left(\Omega t_{\mathrm{eff}}^{\prime} v^{\prime}\right)^{2}} 2 \Omega t_{\mathrm{eff}}^{\prime} v^{\prime}(1+i \mu)\left(\underline{a}_{0}\right)^{2} .
\end{aligned}
$$

Computing the eigenvalues of $\mathbf{M}$ results in

$$
\begin{aligned}
\underline{\lambda}^{\prime} & =\operatorname{Re}\left\{\underline{m}_{A, 1}\right\}+i \operatorname{Re}\left\{\underline{m}_{B, 1}\right\} \pm \underline{\Delta}, \\
\underline{\Delta} & =\left[\left(\operatorname{Re}\left\{\underline{m}_{A, 2}\right\}+i \operatorname{Re}\left\{\underline{m}_{B, 2}\right\}\right)^{2}+\left(\operatorname{Im}\left\{\underline{m}_{A, 2}\right\}+i \operatorname{Im}\left\{\underline{m}_{B, 2}\right\}\right)^{2}-\left(\operatorname{Im}\left\{\underline{m}_{A, 1}\right\}+i \operatorname{Im}\left\{\underline{m}_{B, 1}\right\}\right)^{2}\right]^{1 / 2} .
\end{aligned}
$$

We back substitute $\underline{m}_{A, 1}, \underline{m}_{A, 2}, \underline{m}_{B, 1}, \underline{m}_{B, 2}, \lambda_{i}^{\prime}, \bar{\phi}$, and $\Delta \phi$, and introduce the power of the pumped mode $A=\left|a_{0}\right|^{2}$ to obtain expressions for the real part and the imaginary port of the gain parameter; see Eq. (10) in the main text. From Eq. (A10) we derive the relation between the pump power $F$ and the power of the pumped mode $A$; see Eq. (11) in the main text. 


\section{APPENDIX B: APPROXIMATION OF THE GAIN PARAMETER FOR TECHNICALLY RELEVANT VALUES FOR TPA, FCA, AND PUMP PARAMETERS}

In Sec. IV of the main text we simplify the expression for the real part of the gain parameter $\lambda$ given in Eq. (10) of the main text by assuming technically relevant values for the TPA, FCA, and pump parameters $\left(r \approx 1, \sigma^{\prime} \approx 5, t_{\text {eff }}^{\prime} \approx 0.05-0.5\right.$, $\mu \approx 10, v^{\prime} \approx 200, F \approx 10, A \approx 1$ ) and $\Omega \approx 10$. Here we specify the magnitude of specific terms occurring in Eq. (10) of the main text for the given parameter ranges. We consider two different cases $t_{\text {eff }}^{\prime} \approx 0.05$ (left value below the respective term) and $t_{\text {eff }}^{\prime} \approx 0.5$ (right value below the respective term), and we neglect all terms that are either at least three orders of magnitude smaller than competing terms or that are at least two orders of magnitude smaller and that are approximately constant in $t_{\text {eff }}^{\prime}$. In the following relations, the symbol " $\approx "$ used is to be understood as an order of magnitude quantification rather than as an approximate equality.

$$
\begin{aligned}
& \lambda(\Omega)=-\underbrace{1}_{=1}-\underbrace{2 r A}_{\approx 2}-\underbrace{\sigma^{\prime} r t_{\mathrm{eff}}^{\prime} A^{2}}_{\approx 3 \times 10^{-1}-3 \times 10^{0}} \underbrace{\frac{3+\left(\Omega t_{\mathrm{eff}}^{\prime} v^{\prime}\right)^{2}}{1+\left(\Omega t_{\mathrm{eff}}^{\prime} v^{\prime}\right)^{2}}}_{\approx 1}+\operatorname{Re}\{\underline{\Delta}\},
\end{aligned}
$$

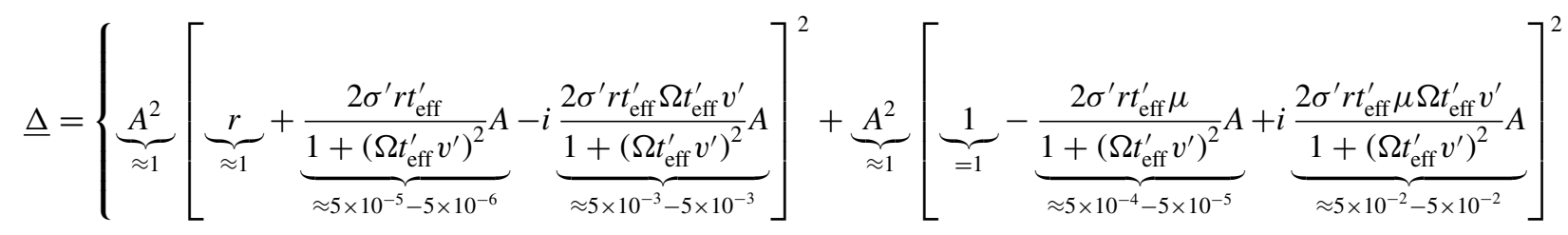

$$
\begin{aligned}
& \left.-[\underbrace{\zeta+d \Omega^{2}-\frac{\phi_{+\Omega}+\phi_{-\Omega}}{2}}_{\text {Arbitrarily large }}-\underbrace{2 A}_{\approx 2}+\underbrace{\sigma^{\prime} r t_{\mathrm{eff}}^{\prime} \mu A^{2}}_{\approx 3 \times 10^{0}-3 \times 10^{1}} \underbrace{\frac{3+\left(\Omega t_{\mathrm{eff}}^{\prime} v^{\prime}\right)^{2}}{1+\left(\Omega t_{\mathrm{eff}}^{\prime} v^{\prime}\right)^{2}}}_{\approx 1}-i \underbrace{\frac{2 \sigma^{\prime} r t_{\mathrm{eff}}^{\prime} \mu \Omega t_{\mathrm{eff}}^{\prime} v^{\prime}}{1+\left(\Omega t_{\mathrm{eff}}^{\prime} v^{\prime}\right)^{2}} A^{2}}_{\approx 5 \times 10^{-2}-5 \times 10^{-2}}]^{2}\right]^{1 / 2} .
\end{aligned}
$$

These approximations are equivalent to approximating the optical field $\underline{a}\left(t^{\prime}, \tau^{\prime}\right)$ generating free carriers by $\left|\underline{a}\left(t^{\prime}, \tau_{\mathrm{S}}^{\prime}+t^{\prime} v^{\prime}\right)\right|^{4} \approx$ $\left|\underline{a}_{0}\right|^{4}$ in Eq. (A6), such that only the first term on the r.h.s. of Eq. (A8) remains. This is consistent with the fact that, at the onset of MI, the instantaneous free-carrier density is still dictated by the unperturbed cw pump. The expression for the real part $\lambda$ of the complex gain parameter then reads

$$
\lambda(\Omega) \approx-1-2 r A-\sigma^{\prime} r t_{\mathrm{eff}}^{\prime} A^{2}+\operatorname{Re}\left\{\sqrt{A^{2} r^{2}+A^{2}-\left(\zeta+d \Omega^{2}-\frac{\phi_{+\Omega}+\phi_{-\Omega}}{2}-2 A+\sigma^{\prime} r t_{\mathrm{eff}}^{\prime} \mu A^{2}\right)^{2}}\right\}
$$

In the absence of $\mathrm{AMC}, \phi_{+\Omega}=\phi_{-\Omega}=0$, this leads to Eq. (12) of the main text.

\section{APPENDIX C: THRESHOLD PUMP POWER FOR MODULATION INSTABILITY: APPROXIMATE ANALYTICAL VS NUMERICAL EVALUATION}

In Fig. 2 of the main text, we analytically evaluate an approximation of the threshold pump power $F_{\text {th }}$ needed to achieve modulation instability, based on realistic values for $t_{\text {eff }}^{\prime}, A, \Omega, r, \sigma^{\prime}, \mu, v^{\prime}$, and for anomalous group-velocity dispersion $d>0$ with $d \Omega_{\max }^{2}-A=0$ at $\lambda\left(\Omega_{\max }\right)=0$. We further assume that mode coupling is absent or sufficiently weak such that avoided mode crossings do not need to be considered $\left(\phi_{\Omega^{\prime}}=0 \forall \Omega^{\prime}\right)$. In the following, we validate this approximation by a numerical investigation. To this end, we find the associated threshold pump power $F_{\text {th,num }}$ by minimizing $F$ according to Eq. (11) of the main text when varying $A, \Omega$, and $\zeta$ under the constraint $\lambda\left(\Omega_{\max }\right)=0$, Eq. (10). We choose $d=0.0025, \mu=7.5, v^{\prime}=189$ and sweep the quantities $r$ and $t_{\mathrm{eff}}^{\prime} \sigma^{\prime}$ to cover the same parameter space as in Fig. 2. Figure 5(a) shows the result of the analytic approximation and corresponds to Fig. 2 of the main text, while Fig. 5(b) displays the numerical results. No difference is visible from the two graphs. The relative difference $\left|\Delta F_{\text {th }}\right| / F_{\text {th,num }}=\left|F_{\text {th }}-F_{\text {th,num }}\right| / F_{\text {th,num }}$ is smaller than $10^{-2}$ in the whole region; see Fig. 5(c).

\section{APPENDIX D: MODE-FIELD SIMULATIONS FOR NONLINEARITY PARAMETER, GROUP REFRACTIVE INDEX, AND DISPERSION PARAMETER OF SILICON-PHOTONIC WAVEGUIDES}

To determine realistic values for the waveguide nonlinearity, the group refractive index, and the dispersion of a microresonator waveguide according to Fig. 1 of the main text, we perform finite-element simulations using commercial tools (RSOFT PHOTONICS CAD suite, FEMSIM). We assume a pump wavelength of $1550 \mathrm{~nm}$ along with the waveguide dimensions specified in Fig. 6. The waveguide in these simulations is either straight or has a bend radius of $115 \mu \mathrm{m}$, corresponding to the resonator design shown in Fig. 1 of the 

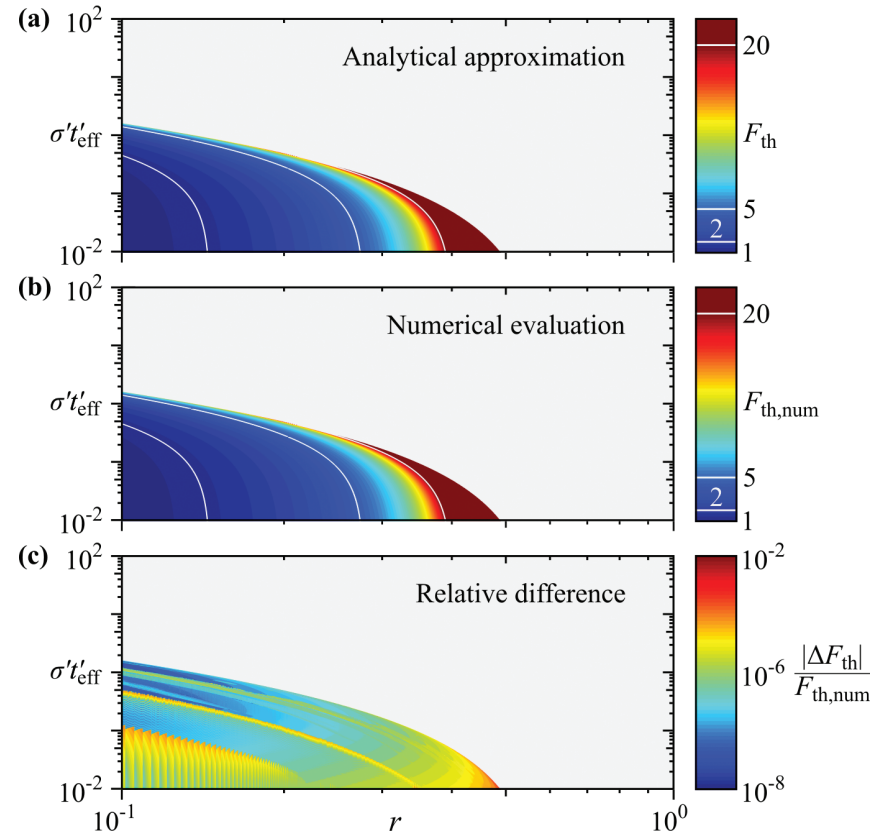

FIG. 5. Threshold pump power needed to achieve modulation instability. (a) Approximate analytical evaluation; see also Fig. 2 of the main text. (b) Corresponding numerical calculation. (c) Relative color-coded difference in threshold pump powers when comparing the analytical and the numerical method. The deviation is smaller than $1 \%$ in the whole region.

main text. The refractive indices of silica $n_{\mathrm{SiO}_{2}}$ and silicon $n_{\mathrm{Si}}$ are included according to Sellmeier's equation [60,61]. The modulus squared of the electric field of the quasi-TE mode field within the silicon waveguide is shown in Fig. 6. The undoped silicon portion of the waveguide is marked with a white outline, and the mode is essentially confined to this region such that the optical field will not be affected by doping associated with the $p-i-n$ - junction or by the vertical interconnect accesses (vias) shown in Fig. 1 of the main text. Since the bend radius $R_{\mathrm{B}} \gg w$ is large relative to the waveguide width $w$ and because $\sqrt{n_{\mathrm{Si}^{2}-n_{\mathrm{SiO}_{2}}^{2}}} R_{\mathrm{B}} \gg \lambda$ holds, the field appears symmetrical in the horizontal direction.

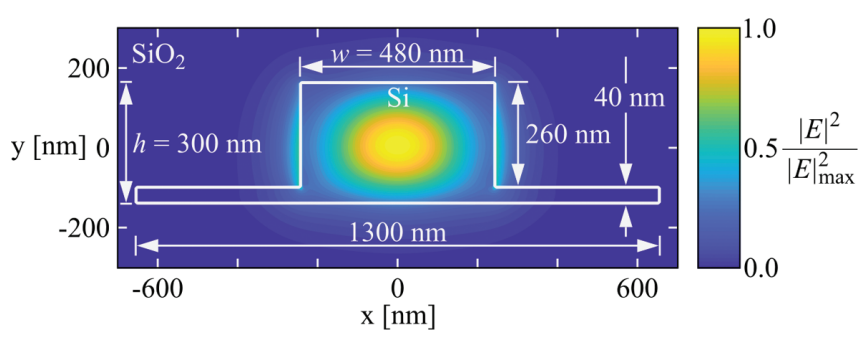

FIG. 6. Normalized modulus squared of the total electric field of the quasi-TE mode at $1550 \mathrm{~nm}$. We assume a waveguide bent to the left with a bend radius of $115 \mu \mathrm{m}$. The white outline marks the undoped silicon part of the waveguide.

Given the field distributions $\mathbf{E}(x, y), \mathbf{H}(x, y)$, we compute the nonlinear waveguide parameter $\gamma$ using [62]

$$
\gamma=\frac{\omega_{\mathrm{P}} \varepsilon_{0} n_{\mathrm{Si}}^{2} n_{2, \mathrm{Si}}}{Z_{0}} \frac{\iint_{\mathrm{Si}}|\mathbf{E}(x, y)|^{4} d x d y}{\left|\iint_{\text {total }} \operatorname{Re}\left\{\mathbf{E}(x, y) \times \mathbf{H}^{*}(x, y)\right\} \cdot \mathbf{e}_{z} d x d y\right|^{2}} .
$$

Here, $\omega_{\mathrm{P}}$ is the angular frequency of the optical pump field, $Z_{0}$ is the free-space wave impedance, and $\mathbf{e}_{z}$ the unit vector along a perimeter of the ring-shaped waveguide. We assume that the third-order nonlinear susceptibility of silicon is a scalar and can be expressed by the corresponding Kerr coefficient $n_{2, \mathrm{Si}}=6.5 \times 10^{-18} \mathrm{~m}^{2} \mathrm{~W}^{-1}$ [20]. The resulting nonlinearity parameter at a wavelength of $1550 \mathrm{~nm}$ amounts to $\gamma=257 \mathrm{~W}^{-1} \mathrm{~m}^{-1}$. In order to determine the dispersion coefficient, mode-field simulations are performed for varying wavelengths in a spectral range of $60 \mathrm{~nm}(7.5 \mathrm{THz})$ around $1550 \mathrm{~nm}$. From these simulations, we obtain the effective refractive index, which is then used to determine the group refractive index $n_{\mathrm{g}}=4.15$ and the dispersion coefficient $\beta_{2}=$ $-0.587 \mathrm{ps}^{2} \mathrm{~m}^{-1}$, both at a wavelength of $1550 \mathrm{~nm}$.

\section{APPENDIX E: INTEGRATION OF THE LUGIATO-LEFEVER EQUATION AND THE FREE-CARRIER EQUATION}

In Sec. V of the main text, we numerically integrate the normalized equations (5) and (6) of the main text using the split-step Fourier method [63]. The normalized temporal (slow) time step is $\Delta t^{\prime}$, the dispersion operator is denoted by $\hat{D}$, and the nonlinear operator is $\hat{N}$ :

$$
\begin{aligned}
\underline{a}\left(t^{\prime}+\Delta t^{\prime}, \tau^{\prime}\right) & =\sqrt{F} \Delta t^{\prime}+e^{\hat{D} \Delta t^{\prime} / 2} e^{\hat{N}\left(t^{\prime}, \tau^{\prime}\right) \Delta t^{\prime}} e^{\hat{D} \Delta t^{\prime} / 2} \underline{a}\left(t^{\prime}, \tau^{\prime}\right), \\
\hat{D} & =-1-i \zeta-i d \frac{\partial^{2}}{\partial \tau^{\prime 2}}, \hat{N}\left(t^{\prime}, \tau^{\prime}\right)=(i-r)\left|\underline{a}\left(t^{\prime}, \tau^{\prime}\right)\right|^{2}-\sigma^{\prime}(1+i \mu) N_{\mathrm{c}}^{\prime}\left(t^{\prime}, \tau^{\prime}-t^{\prime} v^{\prime}\right) .
\end{aligned}
$$

In this approach, the FCA is included in the nonlinear operator $\hat{N}\left(t^{\prime}, \tau^{\prime}\right)$, where the free-carrier density $N_{\mathrm{c}}^{\prime}\left(t^{\prime}, \tau_{\mathrm{S}}^{\prime}\right)$ is computed for each time step $\Delta t^{\prime}$ via Eq. (6). To this end, we use a discretization of $\Delta \tau^{\prime}=\Delta \tau_{\mathrm{S}}^{\prime}=2 \pi / 1024$ for the normalized fast time $\tau^{\prime}$ by dividing the round-trip time into 1024 parts. We further choose the slow-time increment $\Delta t^{\prime}$ as an integer multiple of $\Delta \tau^{\prime} / v^{\prime}$, i.e., $\Delta t^{\prime}=N_{\Delta \tau^{\prime} / v^{\prime}} \Delta \tau^{\prime} / v^{\prime}$ with $N_{\Delta \tau^{\prime} / v^{\prime}}=64$. Over one increment $\Delta t^{\prime}$ of the slow time, the optical field will thus propagate by $N_{\Delta \tau^{\prime} / v^{\prime}}=64$ positions along the fast timescale, which are indexed by an integer $k=0,1, \ldots, 63$ in the following. The evolution of the free carriers during one slow-time increment $\Delta t^{\prime}$ can hence be obtained by integrating Eq. (6) in a recursive manner:

$$
\begin{aligned}
N_{\mathrm{c}}^{\prime}\left(t^{\prime}+(k+1) \frac{\Delta \tau_{\mathrm{S}}^{\prime}}{v^{\prime}}, \tau_{\mathrm{S}}^{\prime}\right) & =N_{\mathrm{c}}^{\prime}\left(t^{\prime}+k \frac{\Delta \tau_{\mathrm{S}}^{\prime}}{v^{\prime}}, \tau_{\mathrm{S}}^{\prime}\right)\left(1-\frac{\Delta \tau_{\mathrm{S}}^{\prime}}{v^{\prime} t_{\mathrm{eff}}^{\prime}}\right)+r \frac{\Delta \tau_{\mathrm{S}}^{\prime}}{v^{\prime}}\left|\underline{a}\left(t^{\prime}, \tau_{\mathrm{S}}^{\prime}+k \Delta \tau_{\mathrm{S}}^{\prime}+t^{\prime} v^{\prime}\right)\right|^{4}, \\
k & =0,1, \ldots, N_{\Delta \tau^{\prime} / v^{\prime}}-1 .
\end{aligned}
$$


Note that, during each slow-time increment $\Delta t^{\prime}$, we neglect the explicit dependence of the optical field $\underline{a}\left(t^{\prime}, \tau^{\prime}\right)$ on the slow time $t^{\prime}$ and only consider the implicit dependence via $\tau^{\prime}=\tau_{\mathrm{S}}^{\prime}+t^{\prime} v^{\prime}$. The delays $t^{\prime} v^{\prime}$ and $k \Delta \tau_{\mathrm{S}}^{\prime}$ of the optical field are implemented as circular rotations of the array associated with $\left|\underline{a}\left(t^{\prime}, \tau_{\mathrm{S}}^{\prime}+k \Delta \tau_{\mathrm{S}}^{\prime}+t^{\prime} v^{\prime}\right)\right|^{4}$. Compared to simplified methods that only consider the evolution of the mean free-carrier density $[17,21]$,

$$
\left\langle N_{\mathrm{c}}^{\prime}\right\rangle_{\tau^{\prime}}\left(t^{\prime}\right)=\frac{1}{2 \pi} \int_{-\pi / 2}^{+\pi / 2} N_{\mathrm{c}}^{\prime}\left(t^{\prime}, \xi\right) d \xi,
$$

the procedure presented here leads to an overall increase of the simulation time by about $10 \%$. To illustrate the capabilities of our model, we perform simulations of the dynamics of an optically pumped Si microresonator and compare the results to those obtained by the mean-value approximation of the intracavity free-carrier distribution [17,21].

In Fig. 7, we show the evolution of the intracavity field power and the free-carrier density by integration of Eqs. (5) and (6) in a regime where free-carrier oscillations occur; see [17] for a discussion of the associated parameter space. Column (a) of Fig. 7 depicts the simulation results obtained by the algorithm following Eqs. (E1) and (E2). The results shown in column (b) are obtained by approximating the free-carrier density by its mean value $\left\langle N_{\mathrm{c}}^{\prime}\right\rangle_{\tau^{\prime}}\left(t^{\prime}\right)$ according to Eq. (E3) and by inserting this value in Eq. (E1). Row R1 shows the simulation parameters, where we assume a significantly increased forcing $F=30$ compared to the cases discussed in the main text. Further, we assume a significantly longer freecarrier lifetime $t_{\text {eff }}^{\prime}=0.406$ corresponding to $122 \mathrm{ps}$ for our assumed microresonator; see Sec. V. Note that the group velocity $v^{\prime}$ is not necessary to compute the mean free-carrier density $\left\langle N_{\mathrm{c}}^{\prime}\right\rangle_{\tau^{\prime}}\left(t^{\prime}\right)$ and is therefore not considered in column (b). Rows R2 and R3 show the evolution of the intracavity (IC) field power $\left|\underline{a}\left(t^{\prime}, \tau^{\prime}\right)\right|^{2}$ and the free-carrier density (FCD) $N_{\mathrm{c}}^{\prime}\left(t^{\prime}, \tau^{\prime}-t^{\prime} v^{\prime}\right)$ as a function of slow time $t^{\prime}$ and as a function of the retarded fast time $\tau^{\prime}$. Both the field power and the carrier density show oscillations with slow time. Modulation instability is absent, and therefore the optical field remains constant along the fast time $\tau^{\prime}$. As a consequence, the free carriers do not show any dependence on $\tau^{\prime}$, independent of the underlying algorithm. In rows R4 and R5, the evolution of the mean values of the optical field power $\left\langle|\underline{a}|^{2}\right\rangle_{\tau^{\prime}}\left(t^{\prime}\right)$ and the carrier density $\left\langle N_{\mathrm{c}}^{\prime}\right\rangle_{\tau^{\prime}}\left(t^{\prime}\right)$ are shown. The vertical gray lines in rows R4 and R5 mark slow-time temporal maxima of the IC power. The maxima of the free-carrier density are delayed relative to the temporal maxima of the optical power, since the free-carrier generation rate $r\left|\underline{a}\left(t^{\prime}, \tau_{\mathrm{S}}^{\prime}+t^{\prime} v^{\prime}\right)\right|^{4}$ in Eq. (6) is larger than the free-carrier decay rate $N_{\mathrm{c}}^{\prime}\left(t^{\prime}, \tau_{\mathrm{S}}^{\prime}\right) / t_{\mathrm{eff}}^{\prime}$ for a certain period, even when the optical field is already declining due to increased FCA. These slow-time dynamics are described in the same manner by both algorithms without any significant difference between the simulation results shown in columns (a) and (b). We therefore conclude that our algorithm correctly describes the slow-time dynamics of the free-carrier density. For another algorithm describing the slow-time dynamics for the fast-time resolved free-carrier density via the boundary condition $N_{\mathrm{c}}\left(t,-t_{\mathrm{RT}} / 2\right)=N_{\mathrm{c}}\left(t+\Delta t,+t_{\mathrm{RT}} / 2\right)$ [22], expressed as $N_{\mathrm{c}}^{\prime}\left(t^{\prime},-\pi\right)=N_{\mathrm{c}}^{\prime}\left(t^{\prime}+\Delta t^{\prime},+\pi\right)$ in normalized quantities, the
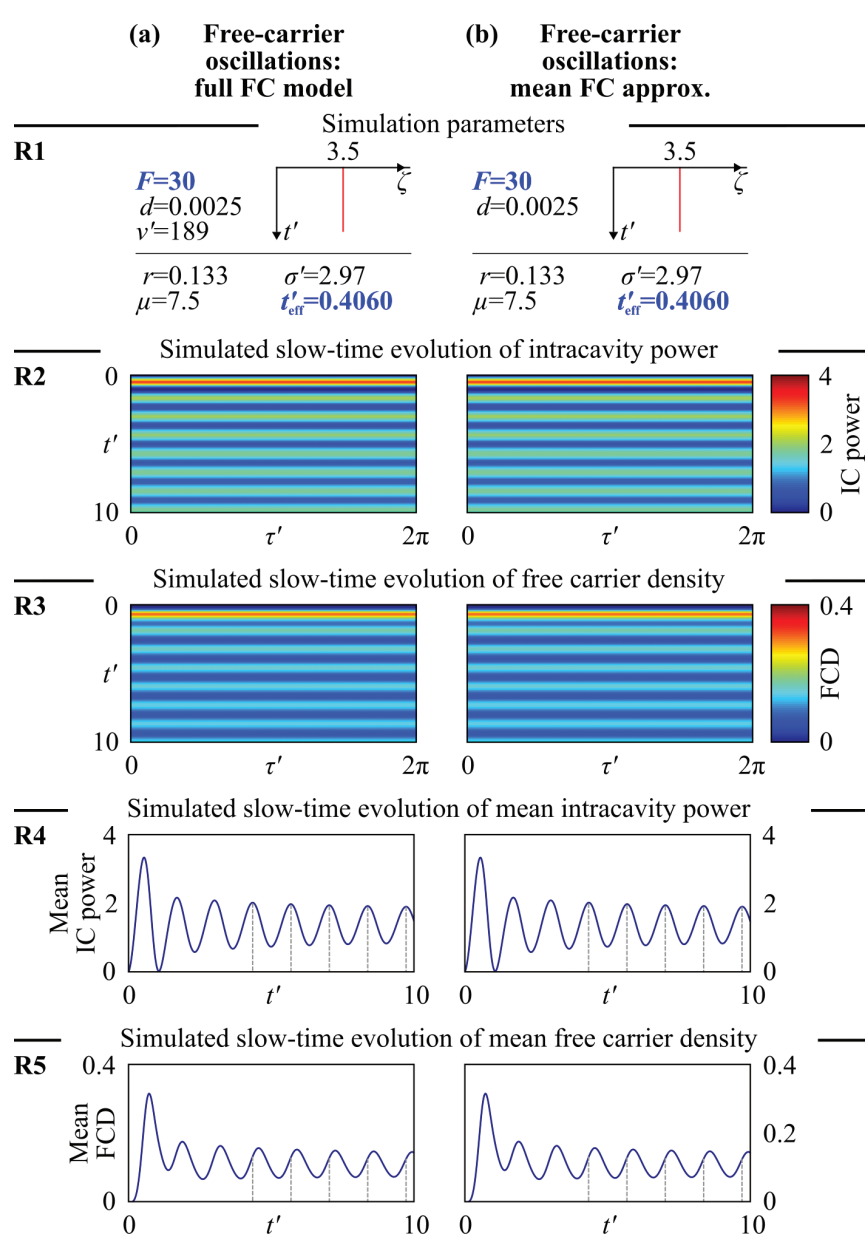

FIG. 7. Results of time integration of the LLE and the freecarrier rate equation for different integration formalisms. Row R1: Simulation parameters. Row R2: Color-coded intracavity (IC) power $\left|\underline{a}\left(t^{\prime}, \tau^{\prime}\right)\right|^{2}$ as a function of fast time $\tau^{\prime}$, evolving over slow time $t^{\prime}$. Row R3: Color-coded free-carrier density (FCD) $N_{\mathrm{c}}^{\prime}\left(t^{\prime}, \tau^{\prime}-t^{\prime} v^{\prime}\right)$ as a function of fast time $\tau^{\prime}$, evolving over slow time $t^{\prime}$. Row R4: Mean IC power $\left\langle\left|\underline{a}\left(t^{\prime}, \tau^{\prime}\right)\right|^{2}\right\rangle_{\tau^{\prime}}$ as a function of the slow time $t^{\prime}$. Vertical dashed lines indicate the temporal positions of maximum intracavity power. Row R5: Mean free-carrier density $\left\langle N_{\mathrm{c}}^{\prime}\left(t^{\prime}, \tau^{\prime}-t^{\prime} v^{\prime}\right)\right\rangle_{\tau^{\prime}}$ as a function of the slow time $t^{\prime}$. Column (a): Free-carrier oscillations simulated using the full model for the description of FC dynamics, Eqs. (E1) and (E2). Column (b): Free-carrier oscillations modeled using a mean free-carrier density. Both models give essentially the same results.

correct accumulation of free carriers is subject to discussion [21].

While the two algorithms give nearly identical results for the IC power and the free-carrier dynamics in the absence of modulation instability, they yield different results when a frequency comb forms. In particular, the dynamics of singlesoliton states on both slow and fast time differ significantly. In Fig. 8, we show the results of a time integration of the LLE and the FCE, Eqs. (5) and (6), for another set of parameters allowing soliton comb formation. Again, column (a) depicts the simulation results obtained through our algorithm, Eqs. (E1) and (E2), while column (b) approximates the free-carrier density through its mean value $\left\langle N_{\mathrm{c}}^{\prime}\right\rangle_{\tau^{\prime}}\left(t^{\prime}\right)$ according to Eq. (E3). 


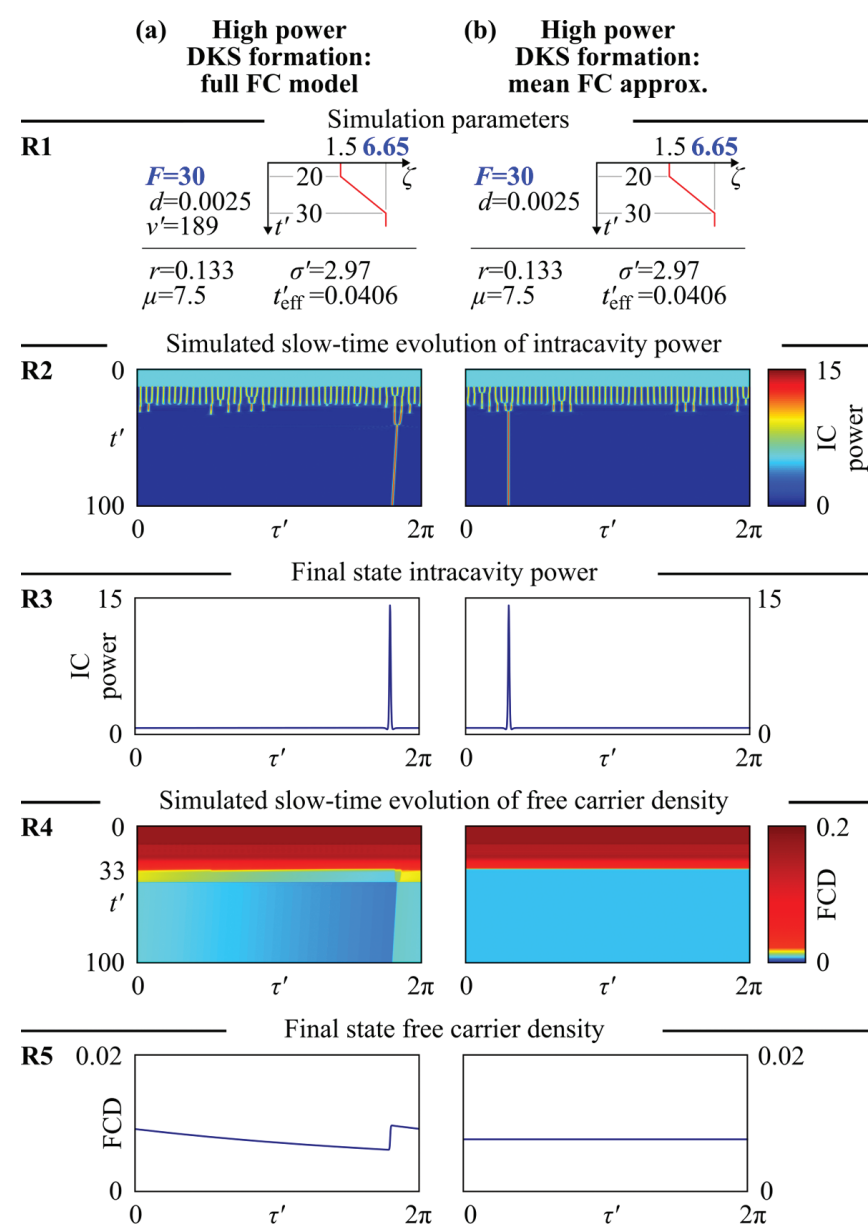

FIG. 8. Results of time integration of the LLE and the freecarrier rate equation for different integration formalisms. Row R1: Simulation parameters. Row R2: Color-coded intracavity (IC) power $\left|\underline{a}\left(t^{\prime}, \tau^{\prime}\right)\right|^{2}$ as a function of fast time $\tau^{\prime}$, evolving over slow time $t^{\prime}$. Row R3: IC power at the end of the simulation, $\left|\underline{a}\left(t^{\prime}=100, \tau^{\prime}\right)\right|^{2}$. Row R4: Color-coded free-carrier density (FCD) $N_{\mathrm{c}}^{\prime}\left(t^{\prime}, \tau^{\prime}-t^{\prime} v^{\prime}\right)$ as a function of fast time $\tau^{\prime}$ evolving over slow time $t^{\prime}$. Row R5: Freecarrier density at the end of the simulation, $N_{\mathrm{c}}^{\prime}\left(t^{\prime}=100, \tau^{\prime}-100 v^{\prime}\right)$. Column (a): Free-carrier oscillations simulated using the full model for the description of FC dynamics, Eqs. (E1) and (E2). Column (b): Free-carrier oscillations modeled using a mean free-carrier density. Correct representation of the fast-time free-carrier dynamics results in a drift of the soliton pulse along the fast time axis $\tau^{\prime}$, which cannot be described by the mean-value approximation of the intracavity free-carrier distribution.

Row R1 lists again the simulation parameters. The forcing is kept at $F=30$, and the detuning is swept until $\zeta=6.65$. The FC lifetime is reduced to $t_{\text {eff }}^{\prime}=0.0406$, corresponding to 12.2 ps. The second row, R2, shows the slow-time evolution of the IC power, where we use different initial random optical fields; see also Sec. V, for columns (a) and (b), respectively. Both algorithms generate a single-soliton pulse around $t^{\prime}=$ 33. In column (a) we observe a drift of the single soliton along the fast-time axis $\tau^{\prime}$ when the fast-time free-carrier dynamics are considered. Such a drift was predicted in another study [19]. In row R4, the evolution of the associated free-carrier density, depicted in the retarded fast time frame $\tau^{\prime}$, is shown. As long as the IC power is high and multiple pulses circulate in the cavity, the FC density is high and nearly constant along $\tau^{\prime}$. However, once only two pulses or only one pulse circulate in the cavity, the free-carrier density drops significantly and is not constant anymore. The fast-time distribution of the free carriers shows the same temporal drift as the optical field. We attribute this drift to the fact that the sharp rise of the free-carrier density during the pulse leads to an attenuation of the trailing edge and hence to an effectively increased propagation velocity. This effect can only be modeled when including the fast-time free-carrier dynamics. The last row, R5, shows the final FC density $N_{\mathrm{c}}^{\prime}\left(t^{\prime}=100, \tau^{\prime}-100 v^{\prime}\right)$. In column (a), the soliton pulse (see row R3) causes a sharp increase of the FC density near $\tau^{\prime} \approx 1.8 \pi$ at $t^{\prime}=100$, such that variations of up to $46 \%$ relative to the mean value of the free carriers are observed during one round trip. These strong variations and the drift of the soliton pulse highlight the importance of the consideration of the fast-time dynamics of the FC density for a more precise description of Kerr comb formation in the presence of TPA and FCA. These effects cannot be described by the mean-value approximation of the intracavity free-carrier distribution; see column (b) of Fig. 8.

Still, despite these inaccuracies, the mean-value approximation of the intracavity free-carrier distribution can give reasonably accurate results for certain device parameters. This is, e.g., the case if the slow-time dynamics of the optical field in the presence of free-carrier absorption such as the drift along the fast time axis $\tau^{\prime}$ are not of interest and if a certain imprecision in the description of the FC density along the microresonator circumference can be tolerated. An upper limit of the fast-time FC density fluctuations, normalized to the mean value of the FC density, can be estimated by assuming that the optical field is represented by a sharp single pulse without a cw background. In this case, the square of the optical power density in the microresonator may be denoted as a single, constant delta pulse, $\left|\underline{a}\left(t^{\prime}, \tau^{\prime}\right)\right|^{4}=A_{\delta}^{2} \delta\left(\tau^{\prime}\right)$, where $\delta\left(\tau^{\prime}\right)$ is the Dirac delta distribution. For simplicity, we assume the pulse to be located at $\tau^{\prime}=0$. In the stationary fast time frame, the optical field then reads $\left|\underline{a}\left(t^{\prime}, \tau_{\mathrm{S}}^{\prime}+t^{\prime} v^{\prime}\right)\right|^{4}=A_{\delta}^{2} \delta\left(\tau_{\mathrm{S}}^{\prime}+t^{\prime} v^{\prime}\right)$. Further assuming that initial FCs do not play a role, $N_{\mathrm{c}}^{\prime}\left(0, \tau_{\mathrm{S}}^{\prime}\right) \approx 0$, and using Eq. (A6), the FC density in the retarded fast time frame reads $N_{\mathrm{c}}^{\prime}\left(t^{\prime}, \tau^{\prime}-v^{\prime} t^{\prime}\right)=N_{\mathrm{c}, 0}^{\prime} \exp \left[-\tau^{\prime} /\left(v^{\prime} t_{\mathrm{eff}}^{\prime}\right)\right]$, where $N_{\mathrm{c}, 0}^{\prime}=r A_{\delta}^{2}\left\{1-\exp \left[-2 \pi /\left(v^{\prime} t_{\mathrm{eff}}^{\prime}\right)\right]\right\}^{-1}$. The maximum value of the FC density along the microresonator circumference is $N_{\mathrm{c}, 0}^{\prime}$, the minimum is given as $N_{\mathrm{c}, 0}^{\prime} \exp \left[-2 \pi /\left(v^{\prime} t_{\mathrm{eff}}^{\prime}\right)\right]$, and the mean value amounts to $\left\langle N_{\mathrm{c}}^{\prime}\left(t^{\prime}, \tau^{\prime}-v^{\prime} t^{\prime}\right)\right\rangle_{\tau^{\prime}}=$ $N_{\mathrm{c}, 0}^{\prime} /(2 \pi) v^{\prime} t_{\mathrm{eff}}^{\prime}\left\{1-\exp \left[-2 \pi /\left(v^{\prime} t_{\mathrm{eff}}^{\prime}\right)\right]\right\}$. Thus the local increase of the FC density caused by the optical pulse, normalized to the mean value $\left\langle N_{\mathrm{c}}^{\prime}\left(t^{\prime}, \tau^{\prime}-v^{\prime} t^{\prime}\right)\right\rangle_{\tau^{\prime}}$ at $\tau^{\prime}=0$, reads

$$
\frac{N_{\mathrm{c}, 0}^{\prime}-N_{\mathrm{c}, 0}^{\prime} \exp \left[-2 \pi /\left(v^{\prime} t_{\mathrm{eff}}^{\prime}\right)\right]}{\left\langle N_{\mathrm{c}}^{\prime}\left(t^{\prime}, \tau^{\prime}-v^{\prime} t^{\prime}\right)\right\rangle_{\tau^{\prime}}}=\frac{2 \pi}{v^{\prime} t_{\mathrm{eff}}^{\prime}}=\frac{t_{\mathrm{RT}}}{t_{\mathrm{eff}}} .
$$

For an FC lifetime equal to the round-trip time, $t_{\mathrm{eff}}=t_{\mathrm{RT}}$, this difference amounts to $100 \%$; i.e., the FC density increase is equal to the mean value along the microresonator circumference. In this case, the mean-value approximation of the intracavity free-carrier distribution may not give reliable 
results. When the lifetime is increased to, e.g., $t_{\mathrm{eff}}=10 t_{\mathrm{RT}}$, the relative FC density increase caused by the optical pulse decreases to $10 \%$ and a mean-FCD approximation becomes increasingly valid. We note that Eq. (E4) is only an approximation that neglects the cw background of the optical field that is usually present-in practice, this aspect will further reduce the fast-time fluctuations of the FC density normalized to its mean value. Thus Eq. (E4) can thus be used as an upper limit for the normalized fast-time FC density fluctuations.

\section{APPENDIX F: DISPERSION PROFILE OF COUPLED MODE FAMILIES}

In this section we describe the derivation of the dispersion profile used in Sec. V of the main text to simulate comb formation in a normal-dispersive microresonator under the influence of an avoided mode crossing; see column (d) of Fig. 3. In a first step, we consider two unperturbed mode families M1 and M2. The comb is generated in the resonances of mode family M1, while mode family M2 may induce local shifts of resonance positions of $\mathrm{M} 1$. The mode families are described by the frequency of the central modes $\omega_{0, \mathrm{M} 1}, \omega_{0, \mathrm{M} 2}$ and by the corresponding free spectral ranges $f_{\mathrm{FSR}, \mathrm{M} 1}, f_{\mathrm{FSR}, \mathrm{M} 2}$, such that the respective resonance frequencies read

$$
\begin{aligned}
\omega_{\Omega^{\prime}, \mathrm{M} 1}= & \omega_{0, \mathrm{M} 1}+\Omega^{\prime}\left(2 \pi f_{\mathrm{FSR}, \mathrm{M} 1}\right) \\
& -\beta_{2} L /\left(2 t_{\mathrm{RT}}\right)\left[\Omega^{\prime}\left(2 \pi f_{\mathrm{FSR}, \mathrm{M} 1}\right)\right]^{2}, \\
\omega_{\Omega^{\prime}, \mathrm{M} 2}= & \omega_{0, \mathrm{M} 2}+\Omega^{\prime}\left(2 \pi f_{\mathrm{FSR}, \mathrm{M} 2}\right) .
\end{aligned}
$$

Here, we have omitted dispersive terms of the second mode family for simplicity. The dispersion profiles of the modes describe the deviation of the various resonance frequencies from an equidistant grid defined by the center free spectral range $f_{\mathrm{FSR}, \mathrm{M} 1}$ of mode family M1. Using Eq. (F1), the dispersion profiles can be stated for the two mode families M1 and M2,

$$
\begin{aligned}
& \omega_{\Omega^{\prime}, \mathrm{M} 1}-\omega_{0, \mathrm{M} 1}-\Omega^{\prime}\left(2 \pi f_{\mathrm{FSR}, \mathrm{M} 1}\right) \\
& \quad=-\beta_{2} L /\left(2 t_{\mathrm{RT}}\right)\left[\Omega^{\prime}\left(2 \pi f_{\mathrm{FSR}, \mathrm{M} 1}\right)\right]^{2}, \\
& \omega_{\Omega^{\prime}, \mathrm{M} 2}-\omega_{0, \mathrm{M} 1}-\Omega^{\prime}\left(2 \pi f_{\mathrm{FSR}, \mathrm{M} 1}\right) \\
& \quad=\omega_{0, \mathrm{M} 2}-\omega_{0, \mathrm{M} 1}-\Omega^{\prime} 2 \pi\left(f_{\mathrm{FSR}, \mathrm{M} 2}-f_{\mathrm{FSR}, \mathrm{M} 1}\right) .
\end{aligned}
$$

The dispersion profile of M1 is a set of discrete points located on a parabola, which is defined by the second-order dispersion coefficient of mode family M1. It allows us to compute the phase deviation $\varphi\left(\Omega^{\prime}\right)$ accumulated by comb modes $\Omega^{\prime}$; see Eq. (16) in the main text. In contrast to the parabolic dispersion profile of mode family $\mathrm{M} 1$, the points given by the dispersion profile of M2 are located on a strongly inclined line, which is indicated in red in Fig. 3(d), row R2 of the main text. Unavoidable deviations from the ideal resonator geometry lead to coupling of the mode families and hence to a hybridization, which is accompanied by a local shift of the resonance frequencies from $\omega_{\Omega^{\prime}, \mathrm{M} 1 / \mathrm{M} 2}$ to two hybrid mode resonances $\omega_{\Omega^{\prime}, \pm}$ for each mode index $\Omega^{\prime}$ [46]. This shift depends on the coupling strength $\theta$ between the respective modes, such that the hybrid mode resonance frequencies are given by [46]

$$
\begin{aligned}
\omega_{\Omega^{\prime}, \pm}= & \frac{\omega_{\Omega^{\prime}, \mathrm{M} 1}+\omega_{\Omega^{\prime}, \mathrm{M} 2}}{2} \\
& \pm \sqrt{\theta^{2}+\frac{\left(\omega_{\Omega^{\prime}, \mathrm{M} 1}-\omega_{\Omega^{\prime}, \mathrm{M} 2}\right)^{2}}{4}} .
\end{aligned}
$$

For these hybrid modes, the dispersion profile is given in the same manner as for $\omega_{\Omega^{\prime}, \mathrm{M} 1 / \mathrm{M} 2}$ by $\omega_{\Omega^{\prime}, \pm}-\omega_{0, \mathrm{M} 1}-$ $\Omega^{\prime}\left(2 \pi f_{\mathrm{FSR}, \mathrm{M} 1}\right)$. Mode family $\mathrm{M} 1$ is the relevant mode family for comb formation in our system. For the gain parameter computation and the simulation of comb formation, we need to choose a certain set of resonances that are close to those of mode family M1 and that contain either the up-shifted frequencies $\omega_{\Omega^{\prime},+}$ or the down-shifted frequencies $\omega_{\Omega^{\prime},-}$ for every mode index $\Omega^{\prime}$. For large differences $\omega_{\Omega^{\prime}, \mathrm{M} 1}-\omega_{\Omega^{\prime}, \mathrm{M} 2}$ of resonance frequencies of the native modes M1 and M2, the native resonance $\omega_{\Omega^{\prime}, \mathrm{M} 1}$ corresponds to the hybrid resonance $\omega_{\Omega^{\prime},+}$ in the case $\omega_{\Omega^{\prime}, \mathrm{M} 1}-\omega_{\Omega^{\prime}, \mathrm{M} 2}>0$ and to the hybrid resonance $\omega_{\Omega^{\prime},-}$ if $\omega_{\Omega^{\prime}, \mathrm{M} 1}-\omega_{\Omega^{\prime}, \mathrm{M} 2}<0$. Near the mode coupling, we choose either $\omega_{\Omega^{\prime},+}$ or $\omega_{\Omega^{\prime},-}$ such that the obtained dispersion profile resembles a typical avoided mode crossing that exhibits approximately symmetric deviations from the native dispersion profile of mode family $\mathrm{M} 1$, as observed in experiments; see, e.g., [46]. From the selected hybrid resonances, we determine the resonance shifts $\delta \omega_{\Omega^{\prime},+}=\omega_{\Omega^{\prime}, \mathrm{M} 1}-\omega_{\Omega^{\prime},+}$

TABLE II. Physical and normalized quantities.

\begin{tabular}{lcccc}
\hline \hline Physical quantity & Symbol & Value & Source & Symbol of equivalent normalized quantity \\
\hline Angular pump frequency & $\omega$ & $2 \pi \times 193.41 \mathrm{THz}$ & Assumption & - \\
Pump power & $P_{\mathrm{in}}$ & $12-50 \mathrm{~mW}$ & Assumption & $F$ \\
Detuning & $\left(\omega_{\mathrm{R}}-\omega_{\mathrm{P}}\right) t_{\mathrm{RT}}$ & $(1.2-2) \mathrm{GHz} \times 2 \pi t_{\mathrm{RT}}$ & Assumption & $\zeta$ \\
Slow time (natural time) & $t$ & - & - & - \\
Fast time (retarded time frame) & $\tau$ & - & - & $t^{\prime}$ \\
Fast time (stationary time frame) & $\tau_{\mathrm{S}}$ & - & - & $\tau^{\prime}$ \\
Normalized group velocity & - & - & - & $\tau_{\mathrm{S}}^{\prime}$ \\
Optical field & $-E$ & - & - & $\underline{a}$ \\
Free-carrier density & $N_{\mathrm{c}}$ & - & - & $N_{\mathrm{c}}^{\prime}$ \\
Side-band frequencies & $\omega_{\Omega^{\prime}}$ & - & - & $\Omega^{\prime}$ \\
Gain rate & - & - & $\underline{\lambda}$ \\
Phase deviations & $\varphi\left(\Omega^{\prime}\right)$ & & $\varphi^{\prime}\left(\Omega^{\prime}\right)$ \\
\hline \hline
\end{tabular}


TABLE III. Microresonator parameters and normalized quantities.

\begin{tabular}{|c|c|c|c|c|c|}
\hline $\begin{array}{l}\text { Microresonator } \\
\text { parameter }\end{array}$ & Symbol & Value & Source & $\begin{array}{c}\text { Symbol of equivalent } \\
\text { normalized quantity }\end{array}$ & $\begin{array}{l}\text { Further occurrence in } \\
\text { formalized quantities }\end{array}$ \\
\hline Power loss per length & $\alpha_{i}$ & $46 \mathrm{~m}^{-1}\left(2 \mathrm{~dB} \mathrm{~cm}^{-1}\right)$ & [20] & - & $d, F, \zeta, t^{\prime}, t_{\mathrm{eff}}^{\prime}, v^{\prime}, \underline{a}, N_{\mathrm{c}}^{\prime}, \phi_{\Omega^{\prime}}, \varphi^{\prime}$ \\
\hline Group refractive index & $n_{\mathrm{g}}$ & 4.15 & Simulation & - & $F, \zeta, \sigma^{\prime}, t^{\prime}, t_{\mathrm{eff}}^{\prime}, v^{\prime}, N_{\mathrm{c}}^{\prime}, \phi_{\Omega^{\prime}}, \varphi^{\prime}$ \\
\hline $\begin{array}{l}\text { Second-order dispersion } \\
\text { coefficient }\end{array}$ & $\beta_{2}$ & $-0.587 \mathrm{ps}^{2} \mathrm{~m}^{-1}$ & Simulation & $d$ & - \\
\hline Nonlinearity parameter & $\gamma$ & $257 \mathrm{~W}^{-1} \mathrm{~m}^{-1}$ & Simulation & - & $F, \underline{a}$ \\
\hline Kerr coefficient & $n_{2}$ & $6.5 \times 10^{-18} \mathrm{~m}^{2} \mathrm{~W}^{-1}$ & {$[20]$} & - & $r, \sigma^{\prime}, N_{\mathrm{c}}^{\prime}$ \\
\hline TPA parameter & $\beta_{\mathrm{TPA}}$ & $0.7 \times 10^{-11} \mathrm{~m} \mathrm{~W}^{-1}$ & {$[20]$} & $r$ & - \\
\hline FC dwell time & $t_{\text {eff }}$ & $12-100 \mathrm{ps}$ & [20] & $t_{\mathrm{eff}}^{\prime}$ & - \\
\hline FC cross section & $\sigma$ & $1.45 \times 10^{-21} \mathrm{~m}^{2}$ & [20] & $\sigma^{\prime}$ & - \\
\hline FC dispersion parameter & $\mu$ & 7.5 & [22] & - & - \\
\hline Confinement factor & $\Gamma_{\mathrm{c}}$ & 1 & Assumption & - & - \\
\hline Round-trip time & $t_{\mathrm{RT}}$ & $10 \mathrm{ps}$ & Assumption & - & $d, F, \zeta, \tau^{\prime}, \tau_{\mathrm{S}}^{\prime}, v^{\prime}, \phi_{\Omega^{\prime}}$ \\
\hline Free spectral range & $f_{\mathrm{FSR}}=t_{\mathrm{RT}}^{-1}$ & $100 \mathrm{GHz}$ & Assumption & - & See round-trip time \\
\hline Local resonance shift & $\delta \omega_{\Omega^{\prime}}$ & Max. $2 \pi \times 875 \mathrm{MHz}$ & Assumption & $\phi_{\Omega^{\prime}}$ & - \\
\hline
\end{tabular}

and $\delta \omega_{\Omega^{\prime},-}=\omega_{\Omega^{\prime}, \mathrm{M} 1}-\omega_{\Omega^{\prime},-}$, respectively. These shifts are used for numeric simulations, the computation of the gain parameter according to Eq. (10) of the main text, and the determination of phase deviations $\varphi^{\prime}\left(\Omega^{\prime}\right)$ according to Eq. (16). Generally, the crucial parameter for modulation instability is the maximum resonance frequency shift $\omega_{\Omega^{\prime}, \mathrm{M} 1}-\omega_{\Omega^{\prime}, \pm}$, which amounts to $-2 \pi \times 875 \mathrm{MHz}$ or approximately $1 \%$ of the FSR of the microresonator under investigation. This is in accordance with experimentally observed resonance shifts [46].

\section{APPENDIX G: LIST OF PHYSICAL QUANTITIES. PARAMETERS FOR THE SIMULATION OF FREQUENCY COMB DYNAMICS}

Table II summarizes both the physical and the normalized quantities used for describing the dynamics of the optical field in the Kerr-nonlinear microresonators. In Table III, we specify physical and normalized microresonator parameters along with their numerical values and the associated source references.
[1] P. Del'Haye, A. Schliesser, O. Arcizet, T. Wilken, R. Holzwarth, and T. J. Kippenberg, Optical frequency comb generation from a monolithic microresonator, Nature 450, 1214 (2007).

[2] T. J. Kippenberg, R. Holzwarth, and S. A. Diddams, Microresonator-based optical frequency combs, Science 332, 555 (2011).

[3] S. B. Papp, K. Beha, P. DelHaye, F. Quinlan, H. Lee, K. J. Vahala, and S. A. Diddams, Microresonator frequency comb optical clock, Optica 1, 10 (2014).

[4] D. T. Spencer, T. Drake, T. C. Briles, J. Stone, L. C. Sinclair, C. Fredrick, Q. Li, D. Westly, B. R. Ilic, A. Bluestone, N. Volet, T. Komljenovic, L. Chang, S. H. Lee, D. Y. Oh, M. G. Suh, K. Y. Yang, M. H. P. Pfeiffer, T. J. Kippenberg, E. Norberg et al., An optical-frequency synthesizer using integrated photonics, Nature 557, 81 (2018).

[5] P. Marin-Palomo, J. N. Kemal, M. Karpov, A. Kordts, J. Pfeifle, M. H. P. Pfeiffer, P. Trocha, S. Wolf, V. Brasch, M. H. Anderson, R. Rosenberger, K. Vijayan, W. Freude, T. J. Kippenberg, and C. Koos, Microresonator-based solitons for massively parallel coherent optical communications, Nature 546, 274 (2017).

[6] M.-G. Suh, Q.-F. Yang, K. Y. Yang, X. Yi, and K. Vahala, Microresonator soliton dual-comb spectroscopy, Science 354, 600 (2016).

[7] M.-G. Suh and K. Vahala, Soliton microcomb range measurement, Science 359, 884 (2018).

[8] P. Trocha, D. Ganin, M. Karpov, M. H. P. Pfeiffer, A. Kordts, J. Krockenberger, S. Wolf, P. Marin-Palomo, C. Weimann, S.
Randel, W. Freude, T. J. Kippenberg, and C. Koos, Ultrafast optical ranging using microresonator soliton frequency combs, Science 359, 887 (2018).

[9] T. Herr, V. Brasch, J. D. Jost, C. Y. Wang, N. M. Kondratiev, M. L. Gorodetsky, and T. J. Kippenberg, Temporal solitons in optical microresonators, Nat. Photonics 8, 145 (2013).

[10] H. Lee, T. Chen, J. Li, K. Y. Yang, S. Jeon, O. Painter, and K. J. Vahala, Chemically etched ultrahigh-Q wedge-resonator on a silicon chip, Nat. Photonics 6, 369 (2012).

[11] M. H. P. Pfeiffer, C. Herkommer, J. Liu, T. Morais, M. Zervas, M. Geiselmann, and T. J. Kippenberg, Photonic damascene process for low-loss, high-confinement silicon nitride waveguides, IEEE J. Sel. Top. Quantum Electron. 24, 6101411 (2018).

[12] X. Chen, M. M. Milosevic, S. Stanković, S. Reynolds, T. D. Bucio, K. Li, D. J. Thomson, F. Gardes, and G. T. Reed, The emergence of silicon photonics as a flexible technology platform, Proc. IEEE 106, 2101 (2018).

[13] D. Thomson, A. Zilkie, J. E. Bowers, T. Komljenovic, G. T. Reed, L. Vivien, D. Marris-Morini, E. Cassan, L. Virot, J. M. Fédéli, J. M. Hartmann, J. H. Schmid, D. X. Xu, F. Boeuf, P. O’Brien, G. Z. Mashanovich, and M. Nedeljkovic, Roadmap on silicon photonics, J. Opt. 18, 073003 (2016).

[14] A. Rickman, The commercialization of silicon photonics, Nat. Photonics 8, 579 (2014).

[15] C. Sun, M. T. Wade, Y. Lee, J. S. Orcutt, L. Alloatti, M. S. Georgas, A. S. Waterman, J. M. Shainline, R. R. Avizienis, S. Lin, B. R. Moss, R. Kumar, F. Pavanello, A. H. Atabaki, H. M. 
Cook, A. J. Ou, J. C. Leu, Y. H. Chen, K. Asanović, R. J. Ram et al., Single-chip microprocessor that communicates directly using light, Nature 528, 534 (2015).

[16] A. G. Griffith, R. K. W. Lau, J. Cardenas, Y. Okawachi, A. Mohanty, R. Fain, Y. Ho, D. Lee, M. Yu, C. T. Phare, C. B. Poitras, A. L. Gaeta, and M. Lipson, Silicon-chip mid-infrared frequency comb generation, Nat. Commun. 6, 6299 (2015).

[17] R. Hamerly, D. Gray, C. Rogers, and K. Jamshidi, Conditions for parametric and free-carrier oscillation in silicon ring cavities, J. Lightwave Technol. 36, 4671 (2018).

[18] R. Haldar, A. Roy, P. Mondal, V. Mishra, and S. K. Varshney, Free-carrier-driven Kerr frequency comb in optical microcavities: Steady state, bistability, self-pulsation, and modulation instability, Phys. Rev. A 99, 033848 (2019).

[19] A. Sahoo and S. Roy, Stability and variational analysis of cavity solitons under various perturbations, Phys. Rev. A 100, 053814 (2019).

[20] A. C. Turner-Foster, M. a Foster, J. S. Levy, C. B. Poitras, R. Salem, A. L. Gaeta, and M. Lipson, Ultrashort free-carrier lifetime in low-loss silicon nanowaveguides, Opt. Express 18, 3582 (2010).

[21] T. Hansson and S. Wabnitz, Dynamics of microresonator frequency comb generation: Models and stability, Nanophotonics 5, 231 (2016).

[22] R. K. W. Lau, M. R. E. Lamont, Y. Okawachi, and A. L. Gaeta, Effects of multiphoton absorption on parametric comb generation in silicon microresonators, Opt. Lett. 40, 2778 (2015).

[23] C. Koos, P. Vorreau, T. Vallaitis, P. Dumon, W. Bogaerts, R. Baets, B. Esembeson, I. Biaggio, T. Michinobu, F. Diederich, W. Freude, and J. Leuthold, All-optical high-speed signal processing with silicon-organic hybrid slot waveguides, Nat. Photonics 3, 216 (2009).

[24] T. Fortier and E. Baumann, 20 years of developments in optical frequency comb technology and applications, Commun. Phys. 2, 153 (2019).

[25] S. Coen, H. G. Randle, T. Sylvestre, and M. Erkintalo, Modeling of octave-spanning Kerr frequency combs using a generalized mean-field Lugiato-Lefever model, Opt. Lett. 38, 37 (2013).

[26] Y. K. Chembo and C. R. Menyuk, Spatiotemporal LugiatoLefever formalism for Kerr-comb generation in whisperinggallery-mode resonators, Phys. Rev. A 87, 053852 (2013).

[27] S. F. Preble, Q. Xu, B. S. Schmidt, and M. Lipson, Ultrafast alloptical modulation on a silicon chip, Opt. Lett. 30, 2891 (2005).

[28] A. G. Griffith, M. Yu, Y. Okawachi, J. Cardenas, A. Mohanty, A. L. Gaeta, and M. Lipson, Coherent mid-infrared frequency combs in silicon-microresonators in the presence of Raman effects, Opt. Express 24, 13044 (2016).

[29] A. C. Turner, C. Manolatou, B. S. Schmidt, M. Lipson, M. A. Foster, Y. Okawachi, and A. L. Gaeta, Tailored anomalous group-velocity dispersion in silicon waveguides, Opt. Express 14, 4357 (2006).

[30] N.-N. Feng, J. Michel, and L. C. Kimerling, Optical field concentration in low-index waveguides, IEEE J. Quantum Electron. 42, 885 (2006).

[31] T. Vallaitis, S. Bogatscher, L. Alloatti, P. Dumon, R. Baets, M. L. Scimeca, I. Biaggio, F. Diederich, C. Koos, W. Freude, and J. Leuthold, Optical properties of highly nonlinear silicon-organic hybrid (SOH) waveguide geometries, Opt. Express 17, 17357 (2009).
[32] C. Koos, P. Vorreau, P. Dumon, R. Baets, B. Esembeson, I. Biaggio, T. Michinobu, F. Diederich, W. Freude, and J. Leuthold, Highly-nonlinear silicon photonics slot waveguide, in Optical Fiber Communication Conference (OFC'08) (Optical Society of America, Washington, DC, 2008), p. PDP25.

[33] C. Bao, Y. Xuan, D. E. Leaird, S. Wabnitz, M. Qi, and A. M. Weiner, Spatial mode-interaction induced single soliton generation in microresonators, Optica 4, 1011 (2017).

[34] X. Xue, M. Qi, and A. M. Weiner, Normal-dispersion microresonator Kerr frequency combs, Nanophotonics 5, 244 (2016).

[35] Y. Liu, Y. Xuan, X. Xue, P.-H. Wang, S. Chen, A. J. Metcalf, J. Wang, D. E. Leaird, M. Qi, and A. M. Weiner, Investigation of mode coupling in normal-dispersion silicon nitride microresonators for Kerr frequency comb generation, Optica 1, 137 (2014).

[36] T. Hansson, D. Modotto, and S. Wabnitz, Dynamics of the modulational instability in microresonator frequency combs, Phys. Rev. A 88, 023819 (2013).

[37] J. Gärtner, R. Mandel, and W. Reichel, The Lugiato-Lefever equation with nonlinear damping caused by two photon absorption, J. Dyn. Diff. Equat. (2021), doi: 10.1007/s10884021-09943-x.

[38] H. K. Tsang and Y. Liu, Nonlinear optical properties of silicon waveguides, Semicond. Sci. Technol. 23, 064007 (2008).

[39] X. Gai, Y. Yu, B. Kuyken, P. Ma, S. J. Madden, J. Van Campenhout, P. Verheyen, G. Roelkens, R. Baets, and B. Luther-Davies, Nonlinear absorption and refraction in crystalline silicon in the mid-infrared, Laser Photonics Rev. 7, 1054 (2013).

[40] Q. Lin, O. J. Painter, and G. P. Agrawal, Nonlinear optical phenomena in silicon waveguides: Modeling and applications, Opt. Express 15, 16604 (2007).

[41] A. D. Bristow, N. Rotenberg, and H. M. Van Driel, Two-photon absorption and Kerr coefficients of silicon for 850-2200 nm, Appl. Phys. Lett. 90, 191104 (2007).

[42] C. Jacoboni, C. Canali, G. Otiaviani, and A. A. Quaranta, A review of some charge transport properties of silicon, Solid. State. Electron. 20, 77 (1977).

[43] W. Bogaerts, P. de Heyn, T. van Vaerenbergh, K. de Vos, S. Kumar Selvaraja, T. Claes, P. Dumon, P. Bienstman, D. van Thourhout, and R. Baets, Silicon microring resonators, Laser Photonics Rev. 6, 47 (2012).

[44] L. Yin and G. P. Agrawal, Impact of two-photon absorption on self-phase modulation in silicon waveguides, Opt. Lett. 32, 2031 (2007).

[45] H. Guo, M. Karpov, E. Lucas, A. Kordts, M. H. P. Pfeiffer, G. Lichachev, V. E. Lobanov, M. L. Gorodetsky, and T. J. Kippenberg, Universal dynamics and deterministic switching of dissipative Kerr solitons in optical microresonators, Nat. Phys. 13, 94 (2017).

[46] Q.-F. Yang, X. Yi, K. Y. Yang, and K. Vahala, Spatial-modeinteraction-induced dispersive-waves and their active tuning in microresonators, Optica 3, 1132 (2016).

[47] X. Xue, Y. Xuan, Y. Liu, P.-H. Wang, S. Chen, J. Wang, D. E. Leaird, M. Qi, and A. M. Weiner, Mode-locked dark pulse Kerr combs in normal-dispersion microresonators, Nat. Photonics 9 , 594 (2015).

[48] C. Milián, A. V. Gorbach, M. Taki, A. V. Yulin, and D. V. Skryabin, Solitons and frequency combs in silica microring 
resonators: Interplay of the Raman and higher-order dispersion effects, Phys. Rev. A 92033851 (2015).

[49] T. Hansson, D. Modotto, and S. Wabnitz, Mid-infrared soliton and Raman frequency comb generation in silicon microrings, Opt. Lett. 39, 6747 (2014).

[50] M. Karpov, H. Guo, A. Kordts, V. Brasch, M. H. P. Pfeiffer, M. Zervas, M. Geiselmann, and T. J. Kippenberg, Raman SelfFrequency Shift of Dissipative Kerr Solitons in an Optical Microresonator, Phys. Rev. Lett. 116, 103902 (2016).

[51] C. Bao, L. Zhang, A. Matsko, Y. Yan, Z. Zhao, G. Xie, A. M. Agarwal, L. C. Kimerling, J. Michel, L. Maleki, and A. E. Willner, Nonlinear conversion efficiency in Kerr frequency comb generation, Opt. Lett. 39, 6126 (2014).

[52] B. Stern, X. Ji, Y. Okawachi, A. L. Gaeta, and M. Lipson, Battery-operated integrated frequency comb generator, Nature 562, 401 (2018).

[53] A. S. Raja, A. S. Voloshin, H. Guo, S. E. Agafonova, J. Liu, A. S. Gorodnitskiy, M. Karpov, N. G. Pavlov, E. Lucas, R. R. Galiev, A. E. Shitikov, J. D. Jost, M. L. Gorodetsky, and T. J. Kippenberg, Electrically pumped photonic integrated soliton microcomb, Nat. Commun. 10, 680 (2019).

[54] M.-G. Suh, C. Y. Wang, C. Johnson, and K. J. Vahala, Directly pumped $10 \mathrm{GHz}$ microcomb modules from low-power diode lasers, Opt. Lett. 44, 1841 (2019).

[55] A. S. Raja, J. Liu, N. Volet, R. N. Wang, J. He, E. Lucas, R. Bouchandand, P. Morton, J. Bowers, and T. J. Kippenberg, Chip-based soliton microcomb module using a hybrid semiconductor laser, Opt. Express 28, 2714 (2020).

[56] T. Horikawa, D. Shimura, and T. Mogami, Low-loss silicon wire waveguides for optical integrated circuits, MRS Commun. 6, 9 (2016).

[57] J. Steinlechner, C. Krüger, N. Lastzka, S. Steinlechner, A. Khalaidovski, and R. Schnabel, Optical absorption measurements on crystalline silicon test masses at $1550 \mathrm{~nm}$, Classical. Quantum Gravity 30, 095007 (2013).

[58] N. M. Wright, D. J. Thomson, K. L. Litvinenko, W. R. Headley, A. J. Smith, A. P. Knights, F. Y. Gardes, G. Z. Mashanovich, R. Gwilliam, and G. T. Reed, Free carrier lifetime modification for silicon waveguide based devices, Opt. Express 16, 19779 (2008).

[59] M. Haelterman, S. Trillo, and S. Wabnitz, Additive-modulationinstability ring laser in the normal dispersion regime of a fiber, Opt. Lett. 17, 745 (1992).

[60] I. H. Malitson, Interspecimen comparison of the refractive index of fused silica, J. Opt. Soc. Am. 55, 1205 (1965).

[61] C. D. Salzberg and J. J. Villa, Infrared refractive indexes of silicon germanium and modified selenium glass, J. Opt. Soc. Am. 47, 244 (1957).

[62] C. Koos, L. Jacome, C. G. Poulton, J. Leuthold, and W. Freude, Nonlinear silicon-on-insulator waveguides for all-optical signal processing, Opt. Express 15, 5976 (2007).

[63] G. Agrawal, Nonlinear Fiber Optics, 5th ed. (Academic Press, New York, 2012). 